\title{
A convergence broadcasting transmission of fixed 4K UHD and mobile HD services through a single terrestrial channel by employing FEF multiplexing technique in DVB-T2
}

\author{
Jong Gyu $\mathrm{Oh}^{1} \cdot$ Yong Ju Won ${ }^{1} \cdot$ Jin Sub Seop Lee ${ }^{1} \cdot$ Joon Tae Kim $^{1}$
}

Received: 6 December 2015 / Accepted: 31 October 2016 / Published online: 9 November 2016

(C) The Author(s) 2016. This article is published with open access at Springerlink.com

\begin{abstract}
Recently, a convergence broadcasting transmission for providing fixed $4 \mathrm{~K}$ ultrahigh-definition (UHD) and mobile high-definition (HD) services through a single terrestrial channel is investigated by employing multiple-physical layer pipe (M-PLP) multiplexing and transmission technologies in digital video broadcasting (DVB)-second-generation terrestrial (T2) systems, and the scalable high-efficiency video coding (SHVC) technique. The M-PLP technique employs different code rates and constellation points for each layer of data and multiplexes differently encoded layers of data into a single frame, with no change of the inverse fast Fourier transform (IFFT) and cyclic prefix (CP). However, the IFFT size should be increased, and the $\mathrm{CP}$ size should be decreased for the 4K UHD layer while the opposite is true for the HD layer. Another aspect is that HD layer data are more important than $4 \mathrm{~K}$ UHD layer data for reliable SHVC decoding, and thus the IFFT size should be decreased and the CP size should be increased for the HD layer to be robust to channel situations. In this paper, the possibility of a terrestrial fixed 4K UHD and mobile HD convergence broadcasting service through a single channel employing the future extension frame (FEF) multiplexing technique is examined. FEF multiplexing technology can be used to adjust the IFFT and CP size for each layer, whereas M-PLP multiplexing technology
\end{abstract}

Joon Tae Kim

jtkim@konkuk.ac.kr

Jong Gyu Oh

riza@konkuk.ac.kr

Yong Ju Won

stonew59@konkuk.ac.kr

Jin Sub Seop Lee

micvanfa@konkuk.ac.kr

1 Department of Electronic Engineering, Konkuk University, Seoul, Republic of Korea cannot. We described the convergence broadcasting service scenario and proposed a transmission system structure by employing FEF and transmission technologies in DVB-T2 systems. Available transmission parameters are extracted and the reception performance of the transmission parameters is examined using computer simulations. From the results, for the 6 and $8 \mathrm{MHz}$ bandwidths, reliable reception of both fixed 4K UHD and mobile HD layer data can be achieved under a static and fast fading multipath channel.

Keywords Digital convergence broadcasting transmission systems · 4K UHD · Mobile HD · FEF · DVB-T2

\section{Introduction}

Worldwide transition from analog to digital broadcasting has now been completed, and the need to study next-generation standards for ultrahigh-definition (UHD) broadcasting, as well as broadcasting and communication convergence systems, is rapidly growing. In November 2011, the future of broadcast television (FoBTV) committee, consisting of 14 organizations including the advanced television systems committee (ATSC), digital video broadcasting (DVB), European broadcasting union (EBU), and electronics and telecommunications research institute (ETRI), was established with a focus on the development of future broadcasting systems. Among these, a consortium of European terrestrial broadcasting service providers, worldwide commercial product makers, and set-top box makers organized themselves as hybrid broadcast broadband television (HbbTV) to develop convergence broadcasting [1,2]. In Japan, hybridcast for the $8 \mathrm{~K}$ super Hi-vision scenario was demonstrated at the NHK 2014 Open House, and a fixed integrated services digital broadcasting (ISDB) — terrestrial (T) and mobile 
one-segment broadcasting services-has been provided in the same radio frequency (RF) channel.

Accordingly, a project that aims to develop convergence broadcasting and monitoring systems that provide optimized and high-quality broadcasting services for both fixed and mobile receivers by employing broadcasting and communication networks was recently initiated in Korea [6,7]. In [6], a convergence broadcasting transmission for providing fixed 4K UHD and mobile HD services through a single terrestrial channel is investigated by employing multiple-physical layer pipe (M-PLP) multiplexing and transmission technologies in DVB-second generation terrestrial (T2) systems [8], and the high efficiency video coding (HEVC)-scalable HEVC (SHVC) technique $[9,10]$. The required data rate when employing the latest SHVC technology was estimated, and the available transmission parameters for $4 \mathrm{~K}$ UHD and HD convergence broadcasting were designed for the proposed transmission systems. The reception performance of the available transmission parameters was then examined by finding the threshold of visibility (TOV).

The M-PLP technique, which was considered in [6], employs different code rates and constellation points for each layer of data and multiplexes differently encoded layers of data into a single frame, with no change of the inverse fast Fourier transform (IFFT) and cyclic prefix (CP), which is used as a guard interval, sizes within a frame. However, the FFT size should be increased, and the CP size should be decreased for the 4K UHD layer, which assumes a static multipath channel with a long delay path, while the opposite is true for the HD layer, which assumes a very fast fading mobile channel. Another aspect is that HD layer data are more important than 4K UHD layer data for reliable SHVC decoding, and thus the IFFT size should be decreased and the $\mathrm{CP}$ size should be increased for the HD layer to be robust to channel situations. Recently, the DVB-T2 Lite profile using future extension frame (FEF) multiplexing technologies has been introduced [8] through which adjustment of the IFFT and CP size for each layer can be achieved. Therefore, both the possibility and the reception performance of a terrestrial $4 \mathrm{~K}$ UHD and HD convergence broadcasting transmission through a single channel is examined by employing the FEF technique instead of the M-PLP technique, which is considered in [6]. In this paper, it is shown that reliable reception of fixed 4K UHD and mobile HD convergence broadcasting can be achieved through 6 and $8 \mathrm{MHz}$ terrestrial channel under targeted channel situations by employing the FEF multiplexing technique.

The rest of the paper is structured as follows. First, the convergence broadcasting service scenario, which can provide fixed $4 \mathrm{~K}$ UHD and mobile HD broadcasting through a single terrestrial channel and (optionally) $8 \mathrm{~K}$ UHD by combining terrestrial broadcasting and communication channels, is described, and transmission requirements for the
SHVC technique are forecast for convergence broadcasting in Sect. 2. In Sect. 3, a convergence broadcasting transmission system for providing fixed 4K UHD and mobile HD services by employing the M-PLP technique and TOVs of its available transmission parameters are briefly summarized. Section 4 presents the FEF multiplexing technique and a convergence broadcasting transmission system structure for fixed 4K UHD and mobile HD services through a terrestrial single channel by employing FEF and transmission technologies in DVB-T2 systems. Section 5 presents available transmission parameters to transmit $4 \mathrm{~K}$ UHD and HD convergence broadcasting through a single 6 and $8 \mathrm{MHz}$ bandwidth channel by employing a convergence broadcasting transmission structure is given. In addition, the reception performance of the available transmission parameters under additive white Gaussian noise (AWGN), static Brazil-D, and time-varying typical urban (TU)-6 channels to find the TOVs. Finally, conclusions and proposals for future work are given in Sect. 6.

\section{Terrestrial fixed 4K UHD and mobile HD convergence broadcasting through a single channel}

\subsection{Terrestrial fixed 4K UHD and mobile HD convergence broadcasting service scenario through a single channel}

In Korea, terrestrial fixed high-definition (HD) broadcasting services are provided by employing ATSC 8-vestigial side band (VSB) systems [3] through $6 \mathrm{MHz}$ bandwidth, while mobile digital multimedia broadcasting (DMB) services are provided using modified Eureak-147 systems [4,5] through $1.536 \mathrm{MHz}$ bandwidth with quarter video graphics array $(\mathrm{QVGA})(320 \times 240)$ resolution, as shown in Fig. 1. However, low-resolution DMB systems do not satisfy consumer needs, and currently viewers prefer mobile broadcasting services based on mobile communication longterm evolution (LTE) systems. In addition, the transmission technologies in ATSC 8-VSB systems were developed in the 1990s. Consequently, the required data rate for UHD broadcasting services may not be achieved through their use. Furthermore, each bandwidth channel is allocated for mobile fixed and broadcasting services. Therefore, convergence broadcasting systems that can provide both terrestrial fixed UHD and high-resolution mobile broadcasting services through a single channel are needed.

Figure 2 shows a conceptual diagram of the terrestrial fixed 4K UHD and mobile HD convergence broadcasting service through a single channel. By employing a scalable video coding (SVC) compression technique, three types of data layers are acquired from original $8 \mathrm{~K}$ UHD video: $8 \mathrm{~K}$ UHD enhanced layer (EL), 4K UHD EL, and HD base layer 

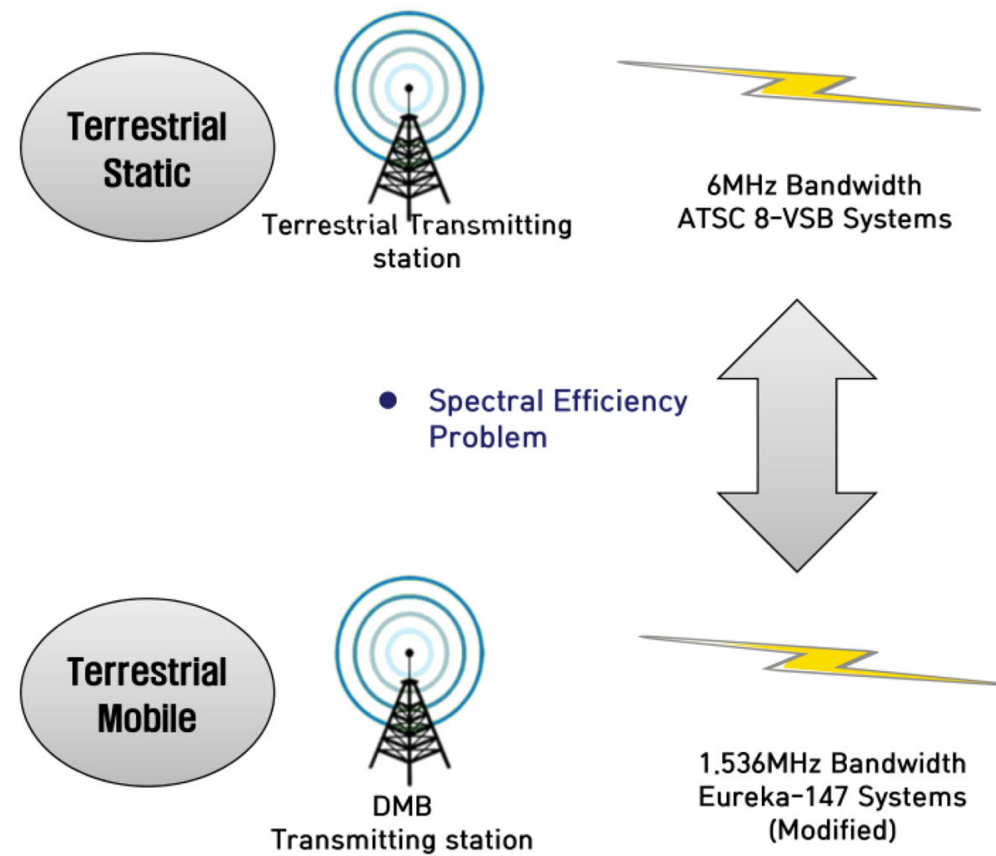

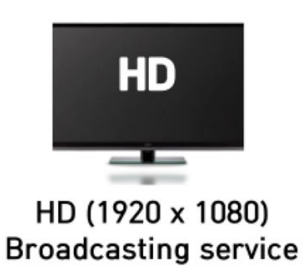

- Transmission capacity lack for UHDTV

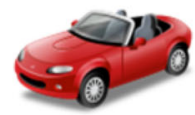

QVGA $(320 \times 240)$

Broadcasting service

- Low Resolution

Fig. 1 Terrestrial fixed HD and mobile DMB broadcasting services, systems, and problems in Korea

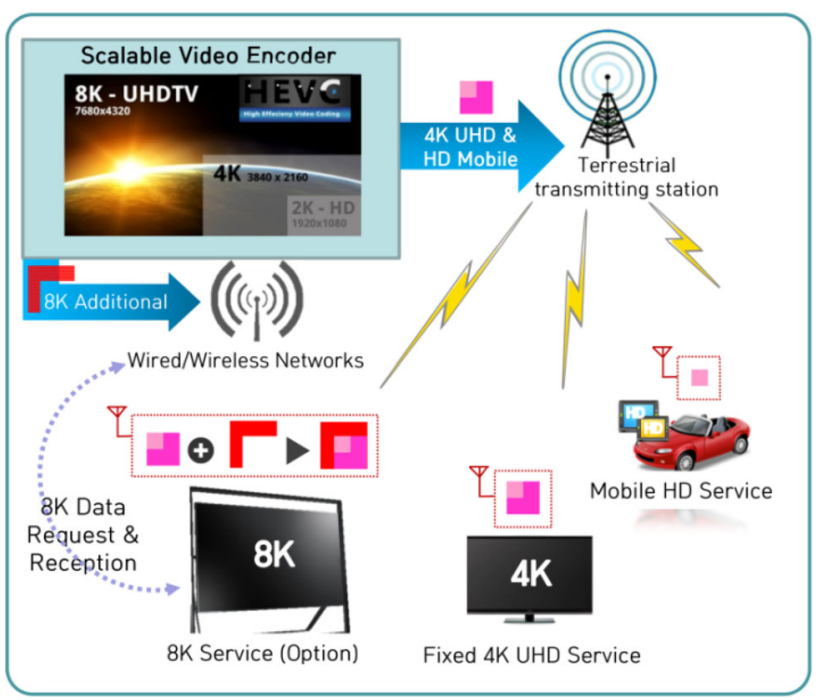

Fig. 2 Conceptual diagram of terrestrial 4K UHD and HD convergence broadcasting service through a single channel

(BL) video data. At terrestrial broadcasting stations, the $4 \mathrm{~K}$ UHD EL and HD BL video data are transmitted through a single channel using convergence broadcasting systems. The mobile viewers then selectively receive HD BL video data, while the fixed receivers get both 4K UHD EL and HD BL video data for $4 \mathrm{~K}$ UHD broadcasting. Optionally, if the fixed receivers are connected to a communication network, $8 \mathrm{~K}$ UHD EL video data from the communication network and 4K UHD EL and HD BL video data from the broadcasting network are combined to provide an $8 \mathrm{~K}$ UHD broadcasting service. In short, a communication network is used for delivering $8 \mathrm{~K}$ UHD additional data, and a broadcasting network is used for delivering HD base and $4 \mathrm{~K}$ UHD additional data. Notice that no mobile network (e.g., long-term evolution, $3 \mathrm{G}$ network) is used for delivering any data. In our study, we focused on convergence broadcasting systems for the transmission of 4K UHD EL and HD BL video data through a terrestrial broadcasting channel.

Therefore, high-quality fixed 4K UHD and mobile HD convergence broadcasting services can be provided instead of conventional fixed HD and mobile DMB broadcasting services through a single terrestrial channel by employing a single transmission system as shown in Fig. 3. From the results, it is evident that high spectral efficiency can be achieved using a single channel and a single transmission system.

\subsection{Video compression technique and transmission requirement for convergence broadcasting}

To examine the possibility of using a terrestrial 4K UHD and HD convergence broadcasting service, the transmission availability of the compressed 4K UHD EL and HD BL data rate through a single terrestrial channel needs to be examined.

The compression ratio of the H.264 video compression technique is $50 \%$ higher than that of the MPEG-2 technique, but the SVC of H.264 shows a reduction in coding efficiency and an increase in problems related to hardware complexity 


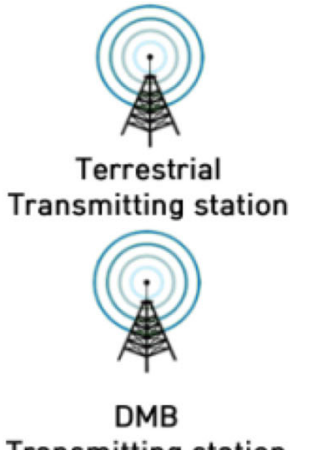

Transmitting station

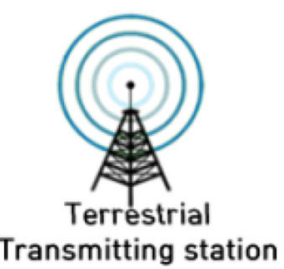

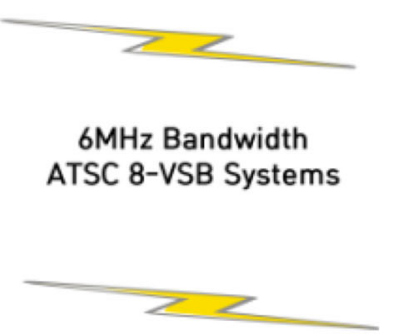

1.536 MHz Bandwidth Eureka-147 Systems (Modified)

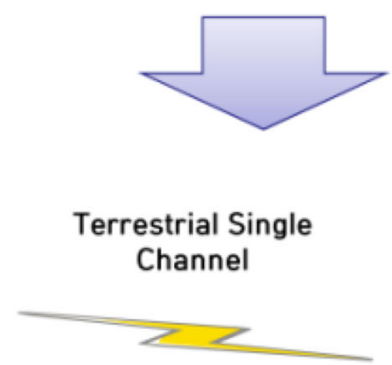

4K UHD \& HD convergence broadcasting systems

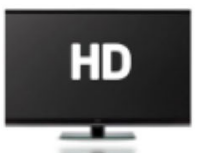

HD (1920 x 1080) Broadcasting service
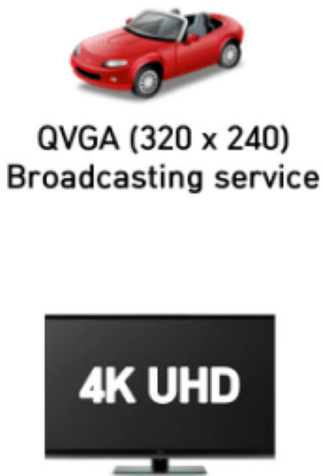

4K UHD (3480 x 2160) Broadcasting service

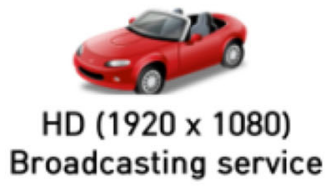

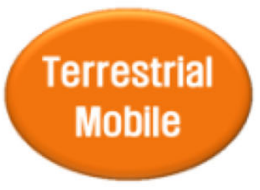

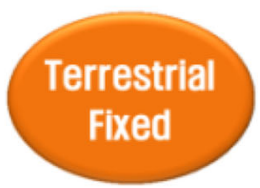

Fixed

Terrestrial

Mobile

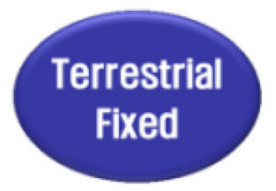

Terrestrial

Mobile

Fig. 3 Service replacement diagram when fixed 4K UHD and mobile HD convergence broadcasting services are provided

[11]. The HEVC technique, for which standardization has been completed recently, is a next-generation video compression technique following $\mathrm{H} .264$ that aims at a maximum compression ratio increase of $50 \%$ over H.264. HEVC can handle the ultrahigh-resolution and picture video content of UHDTV broadcasting times, and employs various techniques such as wide resolution, increased bit depth, losses codec, and scalable coding [9].

Among these techniques, SHVC focuses on lowering implementation complexity to solve the high complexity problem of the scalable coding technique in H.264 [10]. A concept diagram of SHVC is shown in Fig. 4. By employing SHVC, two types of compressed video data are acquired from the original raw video source: UHD EL video data and HD BL video data. While an HD video can be played by decoding HD BL data, a full UHD video can only be played by decoding both UHD EL and HD BL video data via SHVC. Owing to low hardware complexity and high coding efficiency, SHVC is the best choice for the video compression technique of convergence broadcasting services.

As shown in Fig. 5, when considering the worst case scenario and real-time encoding, the compressed data rate when employing the state-of-the-art HEVC encoder is expected to be $25 \mathrm{Mbps}$ for a single $4 \mathrm{~K}$ UHD video and $7 \mathrm{Mbps}$ for a
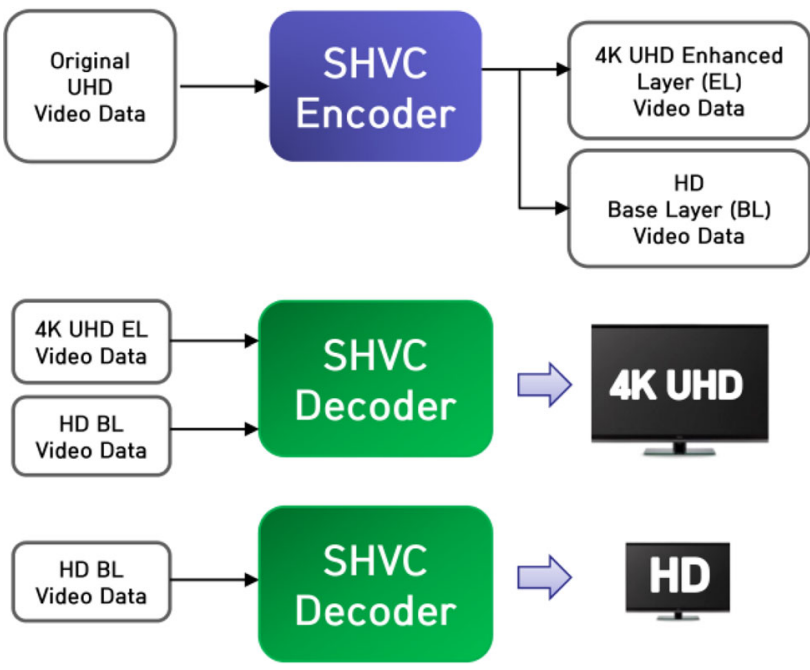

Fig. 4 SHVC encoding and decoding technique concept diagram

single HD video. With the employment of SHVC, the maximum decrease in the rate of approximately $16.5 \%$ [10] and in the average data rate of approximately $15 \%$ is achieved for 4K UHD EL video data compared to single 4K UHD video data. Thus, the compressed data rate would be $21.25 \mathrm{Mbps}$ 


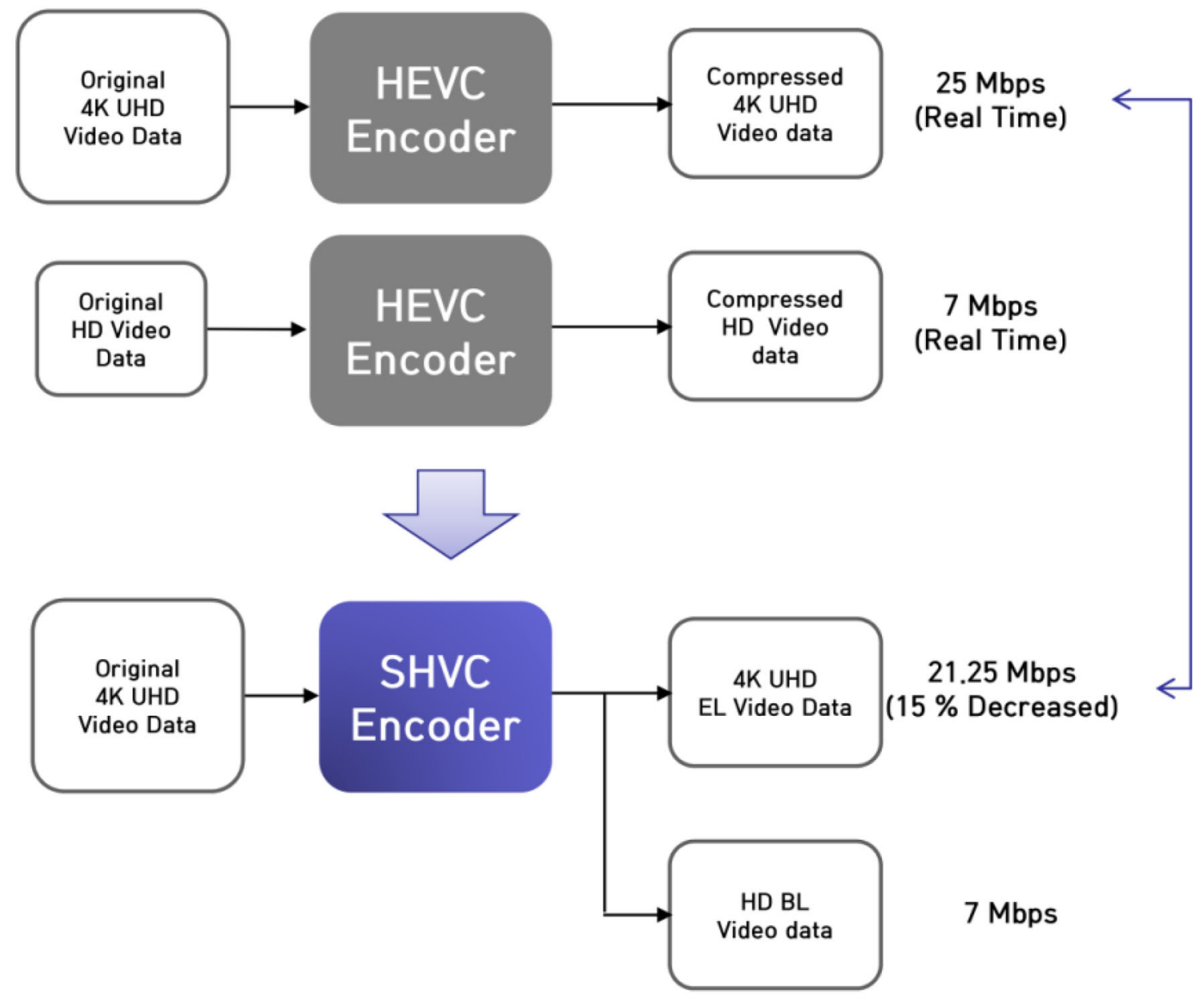

Fig. 5 Required data rate of $4 \mathrm{~K}$ UHD and HD video by employing SHVC

for 4K UHD EL video data, whereas the HD BL video data rate remains the same (i.e., $7 \mathrm{Mbps}$ ). If the performance of a real-time encoder is improved or non-real-time video data are used for transmission, the required data rate used in this study would decrease.

\section{Terrestrial 4K UHD and HD convergence broadcasting transmission through a single channel by employing M-PLP technique}

\subsection{DVB-T2 terrestrial broadcasting transmission systems and M-PLP technique}

The DVB-T2 system [8] is the European second-generation terrestrial digital broadcasting transmission system. Standardization of this system was completed in 2009, and broadcasting services employing DVB-T2 systems are currently in operation in Europe. DVB-T2 systems are based on the orthogonal frequency division multiplexing (OFDM) tech-
Table 1 Transmission modes in DVB-T2 broadcasting transmission systems

\begin{tabular}{ll}
\hline Transmission technology & Transmission mode \\
\hline Channel coding & LDPC \& BCH \\
Code rate & $1 / 2,3 / 5,2 / 3,3 / 4,4 / 5,5 / 6$ \\
Constellation & QPSK, 16QAM, 64QAM, 256 QAM \\
Guard interval & $1 / 4,19 / 256,1 / 8,19 / 128,1 / 16,1 / 32,1 / 128$ \\
FFT size & $1 \mathrm{~K}, 2 \mathrm{~K}, 4 \mathrm{~K}, 8 \mathrm{~K}, 16 \mathrm{~K}, 32 \mathrm{~K}$ \\
Pilot mode & PP1-PP8 \\
\hline
\end{tabular}

nique [12], and adopt the low-density parity check (LDPC) [13] and Bose-Chaudhuri-Hocquenghem (BCH) [14,15] concatenated channel codes, quadrature phase shift keying (QPSK)-256 quadrature amplitude modulation (QAM) constellation, and various sizes of FFT, CP, and PPs (pilot patterns) to maximizing the data transmission capacity, as shown in Table 1. Various interleaving techniques, such as bit, cell, time and frequency interleaving, rotation constellation 


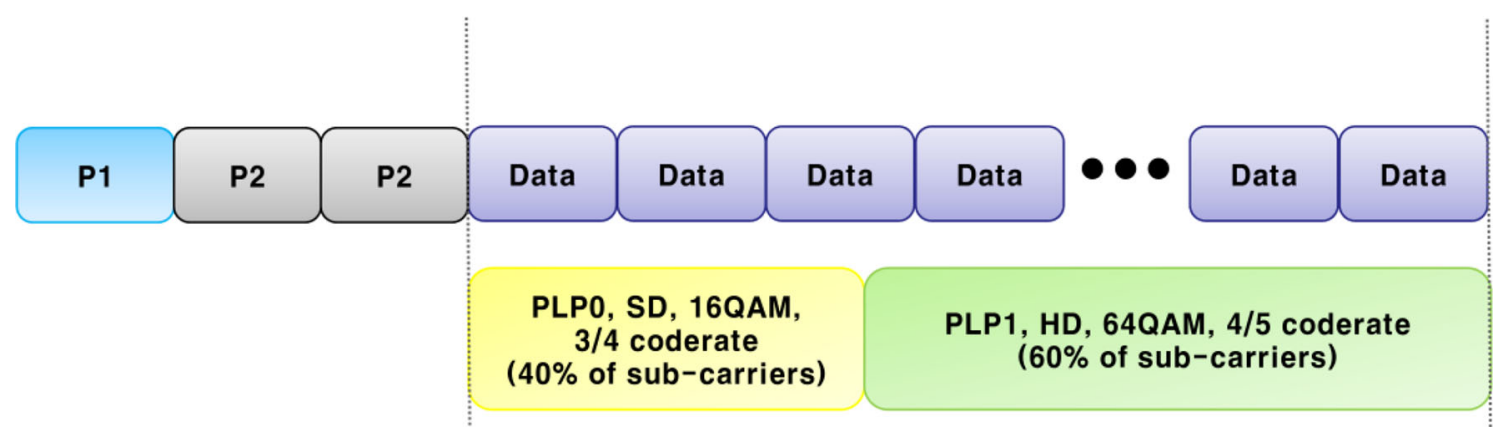

Fig. 6 A DVB-T2 transmission frame structure employing M-PLP technique

scheme, and cyclic Q-delay technique, have been employed in DVB-T2 systems to combat the time-varying channel.

The M-PLP multiplexing technique is employed in DVB$\mathrm{T} 2$ systems to multiplex and transmit multiple layer broadcasting service signals. Different layers of PLP data are encoded and modulated according to each code rate and constellation point, and then multiplexed into a single frame for transmission. For example, PLP 0 data are encoded by the $3 / 4$ code rate and modulated by 16-QAM to provide a standard definition (SD) broadcasting service, while PLP 1 data are encoded by the $4 / 5$ code rate and modulated by 64 -QAM for the HD broadcasting service. M-PLP data are multiplexed into a single frame and transmitted under the same channel conditions as shown. Under the same channel conditions, SD broadcasting data transmitted in the PLP0 layer are more robust against channel errors than HD broadcasting data in PLP 1 owing to the lower code rate and constellation point. Therefore, each receiver selectively acquires PLP layer data depending on the channel conditions.

Figure 6 shows the DVB-T2 transmission frame when the M-PLP technique is employed for the scenario of the previous example, and each block shows an OFDM symbol. The $\mathrm{CP}$ is omitted for convenience, but it is added to all OFDM symbols except the $\mathrm{P} 1$ symbol. The transmission frame is composed of P1, P2, and data OFDM symbols. Note that the FFT and CP sizes of OFDM symbols in a transmit frame do not change, and P1 and P2 symbols are used to carry additional system information. The P1 symbol is positioned at the start of the transmission frame, and its length is always $2048 \times T$ (elementary period [8]) regardless of the FFT size. P2 symbols follow the P1 symbol and are used for carrying system information. The number of P2 symbols $\left(N_{p 2}\right)$ depends on the FFT size as shown in Table 45 of [8], and $N_{p 2}$ is 2 when the FFT size is 8192 . The data OFDM symbols are located after the $\mathrm{P} 1$ and $\mathrm{P} 2$ symbols, and the number of data OFDM symbols $\left(L_{\text {data }}\right)$ is configurable. The number of active subcarriers in an data OFDM symbol $\left(C_{\text {data }}\right)$, which is used for real data transmission, varies depending on the combination of the extension transmission mode, FFT size, and scattered pilot pattern as shown in Table 48 of [8].
A scattered pilot pattern can also be selected by a combination of the FFT and guard interval size as shown in Table 58 of [8]. Thus, $40 \%$ of active subcarriers are used for PLP1 data transmission, and $60 \%$ are used for PLP2 data transmission.

\subsection{Structure of a convergence broadcasting transmission system by employing M-PLP multiplexing technique}

Figure 7 shows a terrestrial single channel fixed 4K UHD and mobile HD convergence broadcasting transmission system structure employing M-PLP multiplexing and transmission technologies in DVB-T2 systems. 4K UHD EL and HD BL video data are acquired by the SHVC encoder, and data of each layer are channel-encoded at different code rates. After channel encoding, data of each layer are interleaved and modulated by each PLP layer. After PLP layer processing, the two sets of PLP data and additional system information data are multiplexed into a single transmission frame by employing the M-PLP multiplexing technique. The single transmission frame is composed of a P1 symbol consisting of 2048 subcarriers; several P2 symbols whose size is dependent on the FFT size of the OFDM symbol; data subcarriers, which are used for transmission of data from the two PLP layers. After the two PLP layers are multiplexed into a single transmission frame, frequency interleaving and OFDM symbol modulation are applied, and then the signals are transmitted after OFDM modulation through a single terrestrial channel. At the receiver side, synchronization and channel equalization are achieved over the OFDM symbol level from the received signal. After frequency deinterleaving, the received frame is demultiplexed into two PLP layers, and decoding is achieved for each layer. The fixed receiver uses data from both decoded layers for 4K UHD video play, while mobile viewers selectively use decoded HD base video data for HD play.

Figure 8 shows the single transmission frame structure when the FFT size is $8192,60 \%$ of active subcarriers are used for transmission of 4K UHD EL data, and $40 \%$ of active subcarriers are used for transmission of HD BL data. The P1 symbol is positioned at the start of the transmission frame, 


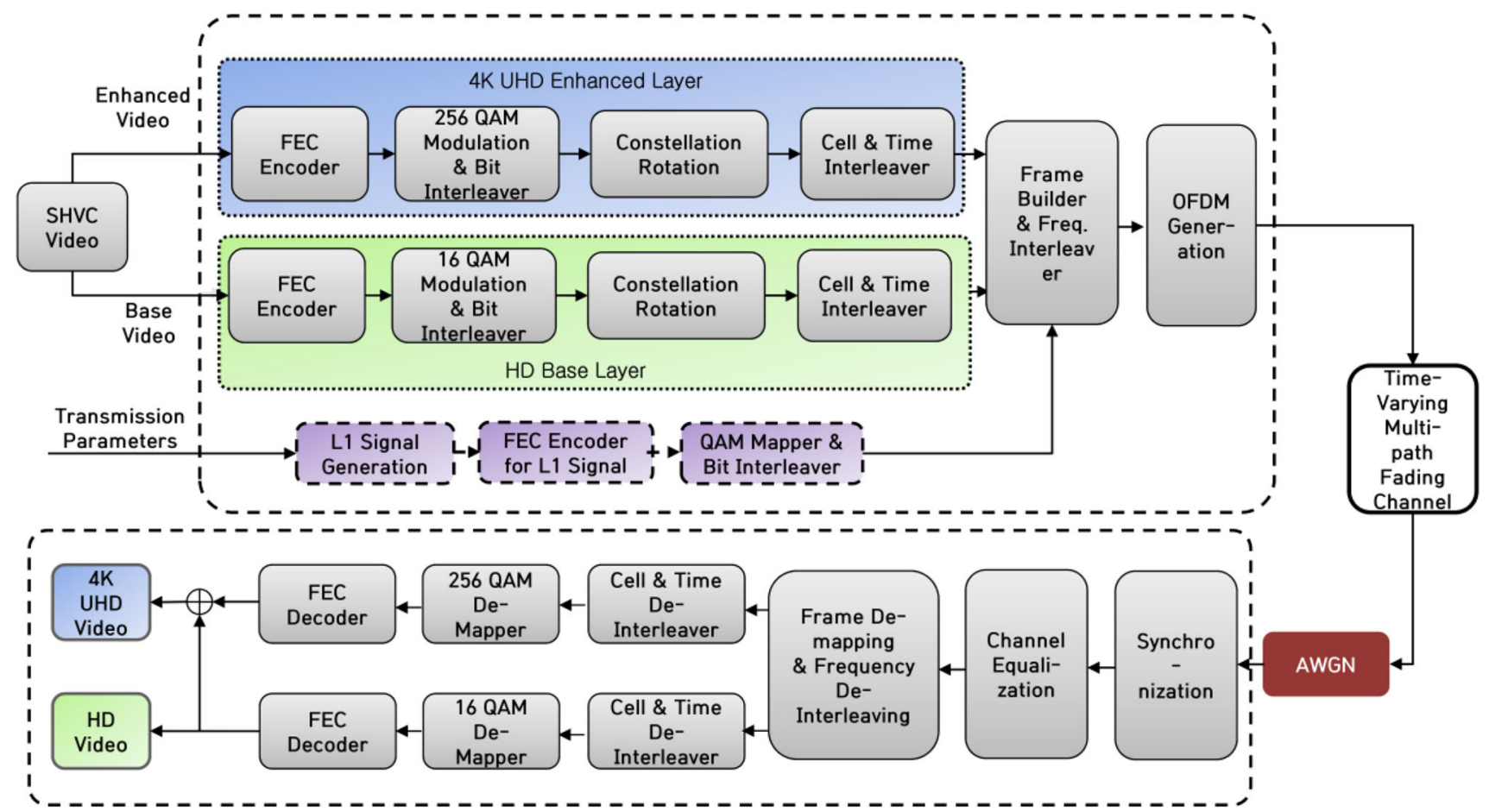

Fig. 7 A structure of the terrestrial $4 \mathrm{~K}$ UHD and HD convergence broadcasting transmission systems through a single channel by employing M-PLP multiplexing technique

Fig. 8 Transmission frame structure for terrestrial $4 \mathrm{~K}$ UHD and HD convergence broadcasting by employing M-PLP multiplexing technique

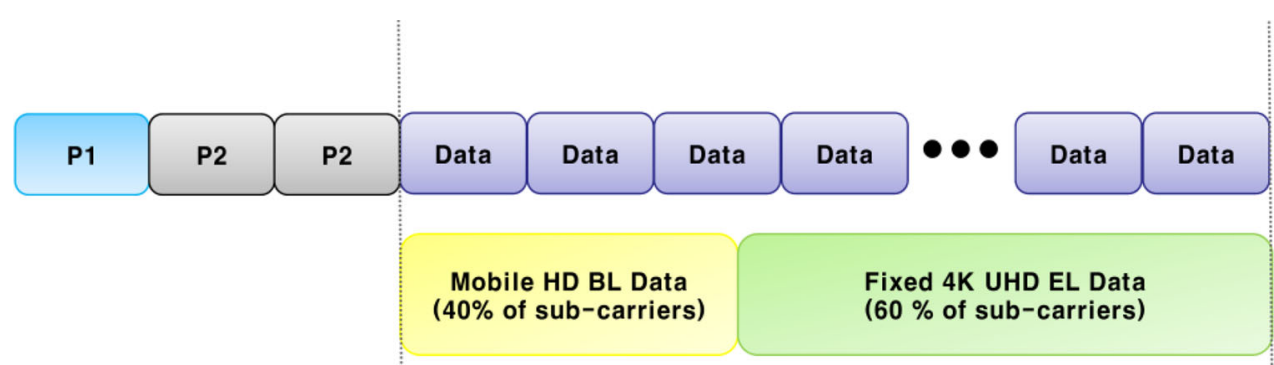

Table 2 Computer simulations environmental conditions

and its length is always $2048 \times T$ regardless of the FFT size. $N_{p 2}$ is 2 when the FFT size is 8192 , and the number of data OFDM symbols is $L_{\text {data }}$.

\subsection{Available transmission parameters and reception performance by computer simulations}

In [6], available transmission parameters obtained by employing the proposed transmission structure are designed for a 6 and $8 \mathrm{MHz}$ bandwidth channel, and the reception performance is analyzed under AWGN and TU-6 channels to find the TOVs. The common computer simulation conditions are listed in Table 2, and the requirements for designing the available transmission parameters are listed in Table 3. The available transmission parameters for each 6 and $8 \mathrm{MHz}$ bandwidth channel and the corresponding TOVs are shown as in Tables 4, 5, 6 and 7, respectively.

From the results in [6], for the $6 \mathrm{MHz}$ bandwidth, reliable reception of HD layer data could be achieved when the

\begin{tabular}{|c|c|}
\hline FEC frame size & 64,800 bits \\
\hline Channel environment & AWGN, and TU-6 channel \\
\hline $\begin{array}{l}\text { Channel estimation method using } \\
\text { pilot symbol }\end{array}$ & Least-square method [16] \\
\hline $\begin{array}{l}\text { Channel interpolation method in } \\
\text { frequency domain }\end{array}$ & $\begin{array}{l}\text { Cubic-spline interpolation method } \\
\text { [17] (the number of pilot symbols } \\
\text { for interpolation: } 12 \text { symbols) }\end{array}$ \\
\hline $\begin{array}{l}\text { Channel interpolation method in } \\
\text { time domain }\end{array}$ & Linear interpolation [18] \\
\hline Center frequency & $\begin{array}{l}476 \mathrm{MHz} \text { (digital TV channel No. } \\
14 \text { in Korea) }\end{array}$ \\
\hline
\end{tabular}

receiver velocity was at a maximum of $140 \mathrm{~km} / \mathrm{h}$ and no higher, owing to the bandwidth limit. When the bandwidth was extended to $8 \mathrm{MHz}$, reliable reception of both 4K UHD and HD layer data was achieved under a very fast fading multipath channel. 
Table 3 Requirements for designing the available transmission parameters

\begin{tabular}{ll}
\hline 4K UHD EL data portion in a frame & $60 \%$ \\
HD BL data portion in a frame & $40 \%$ \\
Maximum length of a frame & $250 \mathrm{~ms} \mathrm{[8]}$ \\
Elementary period $T$ for $6 \mathrm{MHz}$ bandwidth & $7 / 48 \mu \mathrm{s}[8]$ \\
Elementary period $T$ for $8 \mathrm{MHz}$ bandwidth & $7 / 64 \mu \mathrm{s}[8]$
\end{tabular}

If extended transmission mode is available by FFT size, extended transmission mode is applied

Table 4 Available transmission parameters for $6 \mathrm{MHz}$ bandwidth by employing M-PLP multiplexing technique (4K UHD EL layer: 256 QAM, HD BL layer: 16 QAM)

\begin{tabular}{llll}
\hline Parameters & Numbers & & 3 \\
\cline { 2 - 3 } & 1 & 2 & $28,949,373$ \\
\hline Total data rate (bps) & $28,439,336$ & $29,344,584$ & $21,712,029$ \\
4K layer data rate (bps) & $21,329,502$ & $22,234,750$ & $7,237,343$ \\
HD layer data rate (bps) & $7,109,834$ & $7,109,834$ & $5 / 6$ \\
4K layer code rate & $4 / 5$ & $5 / 6$ & $4 / 5$ \\
HD layer code rate & $4 / 5$ & $4 / 5$ & 8,192 \\
FFT size & 8,192 & 8,192 & $1 / 32$ \\
CP size & $1 / 128$ & $1 / 128$ & PP7 \\
Pilot mode & PP7 & PP7 & 200 \\
Number of symbols ${ }^{\mathrm{a}}$ & 200 & 200 & 249.16 \\
Frame length (ms) & 243.51 & 243.51 & Yes \\
Extended mode & Yes & Yes & No \\
Frame closing symbol & No & No & \\
\hline
\end{tabular}

a Number of Data OFDM Symbols in a frame

\section{Terrestrial 4K UHD and HD convergence broadcasting transmission through single channel by employing FEF technique}

While different layers of PLP data are encoded and modulated according to their corresponding code rates and constellation points and then multiplexed into a single frame for transmission by employing the M-PLP multiplexing technique considered in [6], the FFT and CP sizes in a transmission frame may not be changed for each PLP layer.
However, the FFT size should be increased, and the CP size should be decreased for the 4K UHD layer which assumes a static multipath channel with a long delay path, while the opposite is true for the HD layer which assumes a very fast fading mobile channel. Further, HD layer data are more important than 4K UHD layer data for reliable SHVC decoding, and thus the FFT size should be decreased and the CP size should be increased for the HD layer to be robust to channel situations. Recently, the DVB-T2 Lite profile using FEF multiplexing technologies has been introduced [8], through which adjustment of the FFT and CP size for each layer can be achieved. However, the frequencies of the P1 and P2 symbols are increased, and the data transmission efficiency obtained by employing the FEF technique is lower than the efficiency obtained by employing the M-PLP technique. In addition, there are no limitation on the bit rate when employing M-PLP technique and it is the big advantage enabling a flexible service scenario.

\subsection{FEF multiplexing technique and DVB-T2 Lite profile}

The FEF multiplexing technique is adopted to make it possible to extend the DVB-T2 standard. As shown in Fig. 9, a single superframe is composed of periodical parts that contain a number of $\mathrm{T} 2$ frames and a single FEF frame in the FEF technique. In 2011, a DVB-T2 Lite profile obtained by employing FEF was added to the DVB-T2 standard as Annex-I, and conventional T2 transmission details are stored in the T2-Base profile. In the DVB-T2 Lite profile, two additional code rates are added, and the FEC frame size is limited to 16200 bits to take into account a mobile situation. Details of the DVB-T2 Lite profile are given in Table 8.

The usage condition of the constellation rotation technique depending on the combination of the code rate and the constellation point in the DVB-T2 Lite profile is given in Table I.4 of [8], and the scattered pilot pattern depending on the combination of the FFT and the guard interval sizes in the DVB-T2 Lite profile is given in Table I.5 of [8].

Figure 10 shows the superframe structure, which is composed of the T2-Base and T2-Lite transmission frames, and Fig. 11 shows the transmission frame structure of T2-Base

Table 5 TOV of available transmission parameters for $6 \mathrm{MHz}$ bandwidth

\begin{tabular}{|c|c|c|c|c|c|c|}
\hline \multirow{2}{*}{$\begin{array}{l}\text { Parameter } \\
\text { no. }\end{array}$} & \multicolumn{3}{|l|}{ 4K UHD layer } & \multicolumn{3}{|l|}{ HD layer } \\
\hline & AWGN (dB) & $\begin{array}{l}\text { TU-6 }(40 \mathrm{~km} / \mathrm{h}) \\
(\mathrm{dB})\end{array}$ & $\begin{array}{l}\text { TU-6 }(60 \mathrm{~km} / \mathrm{h}) \\
(\mathrm{dB})\end{array}$ & AWGN (dB) & $\begin{array}{l}\text { TU-6 (70 km/h) } \\
(\mathrm{dB})\end{array}$ & $\begin{array}{l}\text { TU-6 (140 km/h) } \\
(\mathrm{dB})\end{array}$ \\
\hline 1 & 20.8 & 26.6 & 30.8 & 9.9 & 15.6 & 20.3 \\
\hline 2 & 21.8 & 28.6 & 41.8 & 9.9 & 15.6 & 20.3 \\
\hline 3 & 21.8 & 28.6 & 41.8 & 10.6 & 16.7 & 31.5 \\
\hline
\end{tabular}


Table 6 Available transmission parameters for $8 \mathrm{MHz}$ bandwidth by employing M-PLP multiplexing technique (4K UHD EL layer: 256 QAM, HD BL layer: 16 QAM)
Table 7 TOV of available transmission parameters for 8 MHz bandwidth

\begin{tabular}{llllll}
\hline Parameters & Numbers & & & & \\
\cline { 2 - 6 } & 1 & 2 & 3 & 4 & 5 \\
\hline Total data rate (bps) & $30,318,345$ & $30,251,372$ & $31,008,536$ & $30,508,286$ & $31,294,731$ \\
4K layer data rate (bps) & $23,101,054$ & $23,039,165$ & $23,919,515$ & $23,245,780$ & $24,140,281$ \\
HD layer data rate (bps) & $7,217,290$ & $7,197,955$ & $7,089,020$ & $7,262,506$ & $7,154,449$ \\
4K layer code rate & $4 / 5$ & $4 / 5$ & $3 / 4$ & $4 / 5$ & $3 / 4$ \\
HD layer code rate & $3 / 4$ & $3 / 4$ & $2 / 3$ & $3 / 4$ & $2 / 3$ \\
FFT size & 1024 & 2048 & 2048 & 4096 & 4096 \\
CP size & $1 / 8$ & $1 / 8$ & $1 / 16$ & $1 / 8$ & $1 / 16$ \\
Pilot mode & PP2 & PP2 & PP4 & PP2 & PP4 \\
Number of symbols ${ }^{\mathrm{a}}$ & 1900 & 950 & 1000 & 475 & 500 \\
Frame length (ms) & 243.51 & 243.51 & 249.16 & 241.64 & 240.128 \\
Extended mode & No & No & No & No & No \\
Frame closing symbol & Yes & Yes & Yes & Yes & Yes \\
\hline N & & & &
\end{tabular}

${ }^{a}$ Number of data OFDM symbols in a frame

\begin{tabular}{lllll}
\hline Parameter no. & 4K UHD layer & & \\
\cline { 2 - 5 } & AWGN $(\mathrm{dB})$ & $\begin{array}{l}\text { TU-6 }(70 \mathrm{~km} / \mathrm{h}) \\
(\mathrm{dB})\end{array}$ & $\begin{array}{l}\text { TU-6 }(140 \mathrm{~km} / \mathrm{h}) \\
(\mathrm{dB})\end{array}$ & $\begin{array}{l}\text { TU-6 }(210 \mathrm{~km} / \mathrm{h}) \\
(\mathrm{dB})\end{array}$ \\
\hline 1 & 20.9 & 26.4 & 26.2 & 26.1 \\
2 & 20.9 & 25.4 & 25.8 & 26.3 \\
3 & 19.5 & 24.2 & 24.0 & 24.3 \\
4 & 20.9 & 26.8 & 27.9 & $\mathrm{X}$ \\
5 & 19.5 & 24.3 & 24.6 & 28.1 \\
\hline Parameter no & HD layer & & & \\
\cline { 2 - 5 } & AWGN $(\mathrm{dB})$ & TU-6 $(70 \mathrm{~km} / \mathrm{h})$ & $\mathrm{TU}-6(140 \mathrm{~km} / \mathrm{h})$ & $\mathrm{TU}-6(210 \mathrm{~km} / \mathrm{h})$ \\
& & $(\mathrm{dB})$ & $(\mathrm{dB})$ & 14.1 \\
\hline 1 & 9.0 & 16.0 & 13.8 & 13.9 \\
2 & 9.0 & 13.9 & 14.4 & 11.4 \\
3 & 7.6 & 12.9 & 12.3 & 14.5 \\
4 & 9.0 & 14.2 & 13.9 & 12.2 \\
\hline
\end{tabular}

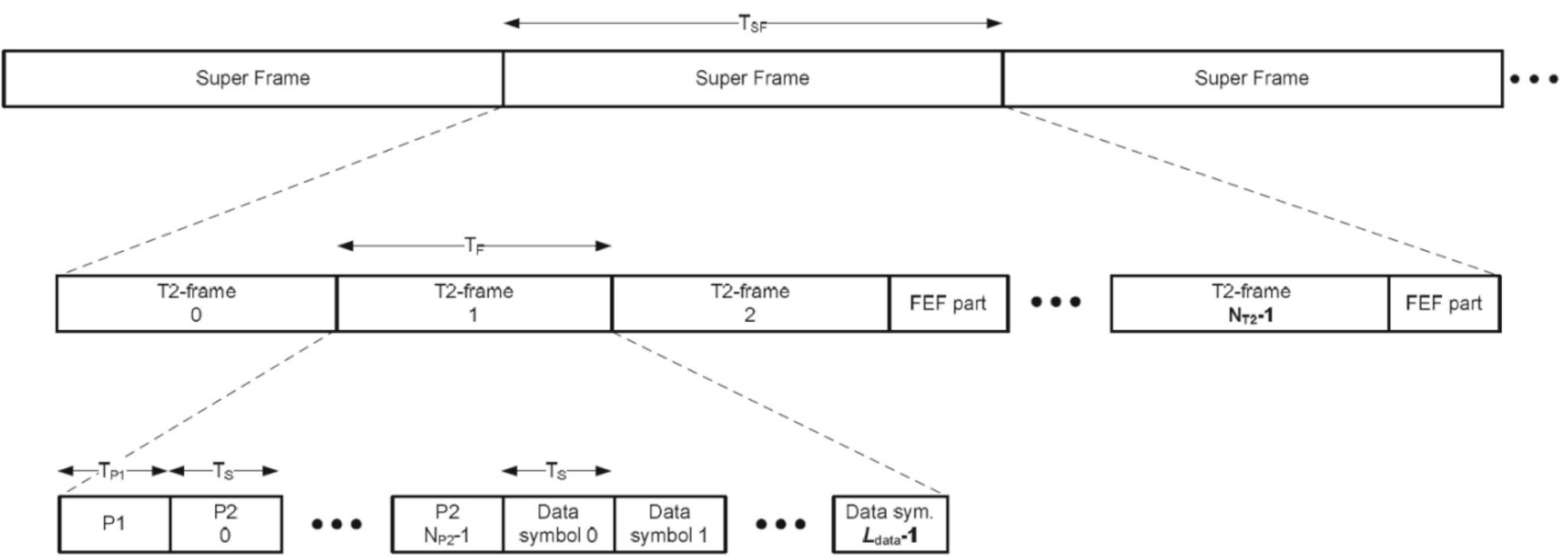

Fig. 9 A DVB-T2 transmission frame structure employing FEF technique 
and T2-Lite profile data, where each block shows an OFDM symbol. The $\mathrm{CP}$ is omitted for convenience, but it is added to all OFDM symbols except the P1 symbol. The FFT size for T2-Base is 32,768 and that for T2-Lite is 2048. The number of consecutive T2-Base frames in a single T2-Base superframe (T2-Base FEF_INTERVAL) is 3, and the number of consecutive T2-Lite frames in a single T2-Lite superframe (T2-lite FEF_INTERVAL) is 1. Each Base and Lite superframe must be composed of more than two Base or Lite frames, the P1 symbol is positioned at the start of the transmission frame, and its length is always $2048 \times T$ regardless of the FFT size. P2 symbols occur after the P1 symbol and are used for car-

Table 8 Transmission modes and technologies in DVB-T2 Lite profile

\begin{tabular}{ll}
\hline FEC & LDPC and BCH, 16,200 bits only \\
\hline $\begin{array}{l}\text { Channel code } \\
\text { rate }\end{array}$ & $1 / 3,2 / 5,1 / 2,3 / 5,2 / 3,3 / 4$ \\
Constellation & QPSK, 16QAM, 64QAM, 256QAM \\
Guard interval & $1 / 4,19 / 256,1 / 8,19 / 128,1 / 16,1 / 32,1 / 128$ \\
FFT size & $2 \mathrm{~K}, 4 \mathrm{~K}, 8 \mathrm{~K}, 16 \mathrm{~K}$ \\
Pilot mode & PP1-PP7 \\
$\begin{array}{l}\text { Maximum } \\
\text { T2-Lite frame } \\
\text { length }\end{array}$ & $1 \mathrm{~S}(250 \mathrm{~ms}$ for T2-Base profile $)$ \\
\hline
\end{tabular}

rying system information. The numbers of Base $\mathrm{P} 2$ symbols $\left(N_{p 2 \_b a s e}\right)$ and Lite P2 symbols $\left(N_{p 2 \_l i t e}\right)$ depend on the FFT sizes of each profile as in Table 2. Thus, $N_{p 2 \_b a s e}$ is 1 and $N_{p 2 \_l i t e}$ is 8 . Data OFDM symbols occur after the P2 symbols, depending on the number of data OFDM symbols of each profile ( $L_{\text {data_base }}$ and $\left.L_{\text {data_lite }}\right)$.

Base and Lite signals of the foregoing frame structure can be acquired selectively at the receiver by decoding the $\mathrm{S} 1$ field information carried by the P1 symbol, as given in Table 18 of [8]. Fixed and mobile receivers first decode the P1 symbol of each transmission frame, and selectively receive a signal appropriate for their channel situation.

\subsection{Terrestrial 4K UHD and HD convergence broadcasting transmission system through single channel by employing FEF multiplexing technique}

In Sect. 2.2 and [6], the estimated data rates for transmitting 4K UHD EL data and HD BL data are 25.25 Mbps and 7 Mbps, respectively, for convergence broadcasting through a single terrestrial channel. However, when the M-PLP multiplexing technique is employed for convergence broadcasting systems in [6], the minimum FFT size of the available transmission parameters for a $6 \mathrm{MHz}$ bandwidth channel is restricted to 8192 , and code rates for the two layers are

Fig. 10 A superframe structure that is composed of T2-Base and Lite transmission frames
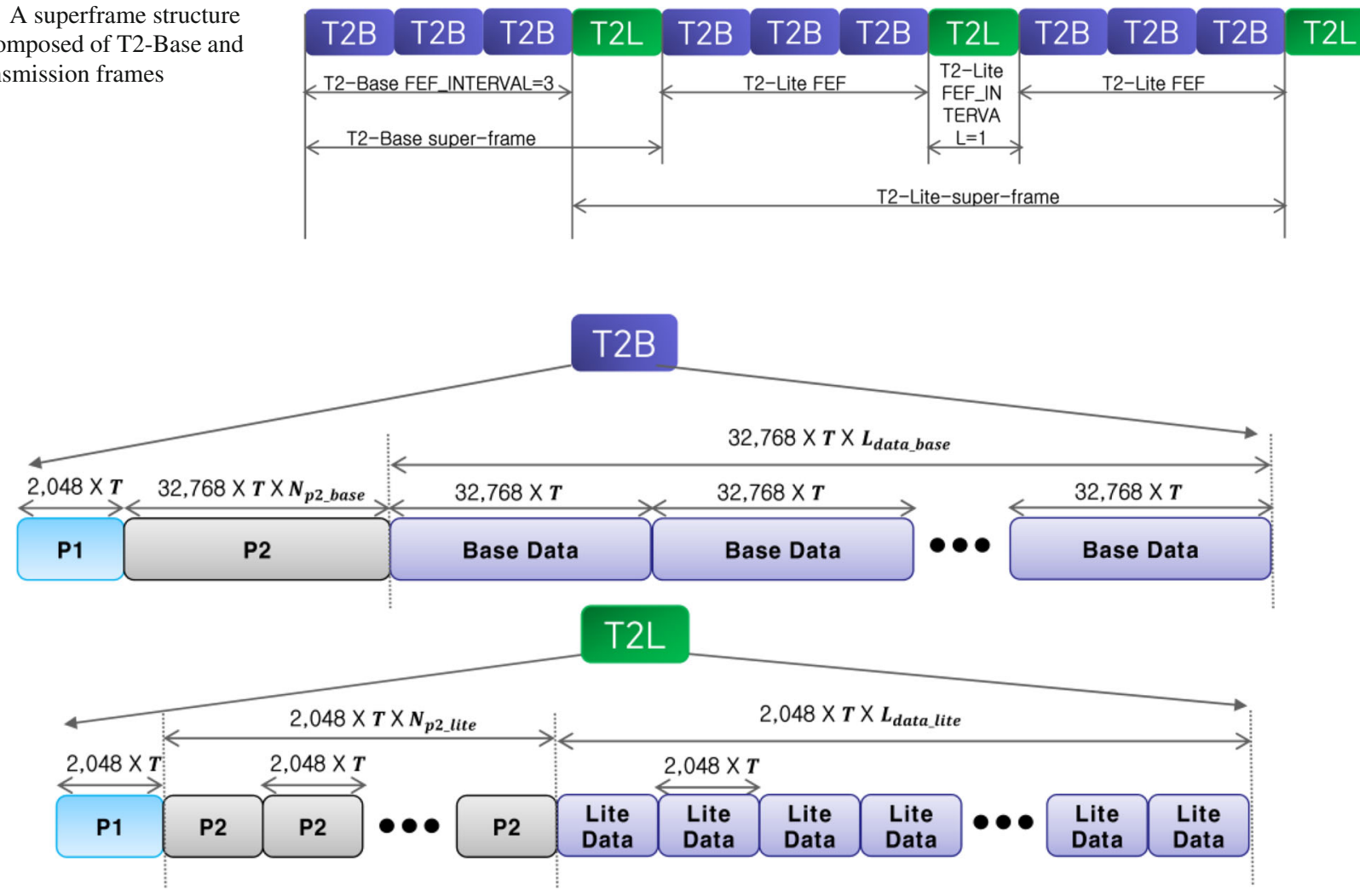

Fig. 11 A transmission frame structure of T2-Base and T2-Lite profile data 
Table 9 Required transmission data rate of each 4K UHD and HD layer for 6 and $8 \mathrm{MHz}$ bandwidth

\begin{tabular}{lll}
\hline $\begin{array}{l}\text { Bandwidth } \\
(\mathrm{MHz})\end{array}$ & Layer & \\
\cline { 2 - 3 } & 4K UHD layer & HD layer \\
\hline 8 & $21.25 \mathrm{Mbps}$ & $7 \mathrm{Mbps}$ \\
6 & $\begin{array}{l}18.0625 \mathrm{Mbps}(15 \% \\
\text { decreased than the data } \\
\text { rate in } 8 \mathrm{MHz})\end{array}$ & $\begin{array}{c}\text { 5.95 Mbps }(15 \% \\
\text { decreased than the } \\
\text { data rate in } 8 \mathrm{MHz})\end{array}$ \\
\hline
\end{tabular}

somewhat high. In addition, the data transmission efficiency obtained by employing the FEF technique is rather low compared to that obtained by employing the M-PLP technique. As a result, it is difficult to design the available transmission parameter that can achieve the estimated data rates in Sect. 2.2 by employing the FEF technique for $6 \mathrm{MHz}$ bandwidth. Thus, in this study, we design available transmission parameters for different required data rates for each 6 and 8 $\mathrm{MHz}$ bandwidth channel. Lowering the data rate can degrade video quality, but the data rate for a $6 \mathrm{MHz}$ bandwidth channel is decreased by $15 \%$ compared to the estimated data rate in Sect. 2.2 by considering of lowering sampling rate, frame rate and improving real-time SHVC encoder performance. For an $8 \mathrm{MHz}$ bandwidth channel, the estimated data rate in Sect. 2.2 is applied. The estimated data rates are summarized in Table 9.

Meanwhile, there is no data rate restriction in using FEF multiplexing technique, the DVB-T2 Lite profile restricts a maximum data rate to $4 \mathrm{Mbit} / \mathrm{s}$ [8]. Therefore, the estimated data rate in this paper may not meet the requirement of DVB-T2 Lite profile. However, in this paper, we focus on examining the possibility and the reception performance of a terrestrial $4 \mathrm{~K}$ UHD and HD convergence broadcasting transmission through a single channel by employing the FEF multiplexing technique and transmission technology in DVB-T2 systems (Lite profile is included in DVB-T2 systems), and do not restrict the purpose of our study to following DVB-T2 Lite profile. In addition, if the performance of a realtime encoder is improved or non-real-time video data are used for transmission, the estimated data rate used would decrease and may meet the requirement of DVB-T2 Lite profile.

Figure 12 shows a terrestrial single channel fixed $4 \mathrm{~K}$ UHD and mobile HD convergence broadcasting transmission system structure that employs FEF multiplexing and transmission technologies in DVB-T2 systems. The acquired 4K UHD EL data from SHVC are channel-encoded, mapped onto constellations, and bit-, cell-, and time-interleaved depending on the base profile. Acquired HD BL data are similarly processed depending on the Lite profile. Further, layer data are modulated, and a transmission frame is composed depending on different FFT and CP sizes, pilot pattern, the number of data OFDM symbols in a frame, etc., in accordance with each layer profile. After each transmission frame is composed, a superframe is made of transmission frames of each layer by T2-Base FEF_INTERVAL and T2lite FEF_INTERVAL FEF, and is transmitted through a single terrestrial channel.

A fixed receiver acquires a signal under a static multipath channel and first decodes a P1 symbol to get the S1 field information. Using this information, the transmitted superframe is demultiplexed into each layer transmission frame. Each layer transmission frame is decoded by each profile (which is acquired by decoding the P1 and P2 symbols), and the fixed receiver uses data from both decoded layers for the 4K UHD video play. On the other hand, a mobile receiver acquires a signal under a fast-fading multipath channel, and only transmission frames of the HD BL layer are demultiplexed from the superframe using decoded S1 field information. A mobile receiver decodes transmission frames of the HD BL layer from the Lite profile, and mobile viewers selectively use the decoded HD base video data for HD play. Synchronization and channel equalization are commonly achieved over the OFDM symbol of each layer transmission frame level.

\section{Available transmission parameters and reception performances by computer simulations}

On the basis of the estimated data rate from SHVC in Sect. 2 and employing the transmission systems described in Sect. 3, available transmission parameters are designed for transmission of convergence broadcasting of two-layer data through a single 6 and $8 \mathrm{MHz}$ bandwidth channel. Simultaneously the reception performance of each parameter was analyzed using computer simulations under AWGN, static Brazil-D, and TU-6 channels.

The data rate of each layer depends on the transmission mode (constellation point, code rate, FFT and CP size, pilot mode, the number of subcarriers, the number of active subcarriers, the portion of system information, etc.) [8]. The available parameters for $6 \mathrm{MHz}$ bandwidth to meet the requirements in Table 10 are shown in Table 11.

In DVB-T2 systems, the number of cases of transmission parameter combinations (e.g., constellation, code rate, FFT size, and pilot pattern, etc.) are numerous. Thus, we first set the conditions of Table 10, and found the combination of transmission parameters using a computer program loop. When using a computer program loop, every combination of the constellation, code rate, FFT size, CP size, pilot pattern, number of data OFDM symbols in a frame, FEF Interval for each 4K UHD BL and HD BL were changed, and bit rates of each layer were examined whether to meet the required data rate in Table 9.

As the results, it was found that the required data rate (Sect. 2.2) could be met when 256 QAM was used for $4 \mathrm{~K}$ 


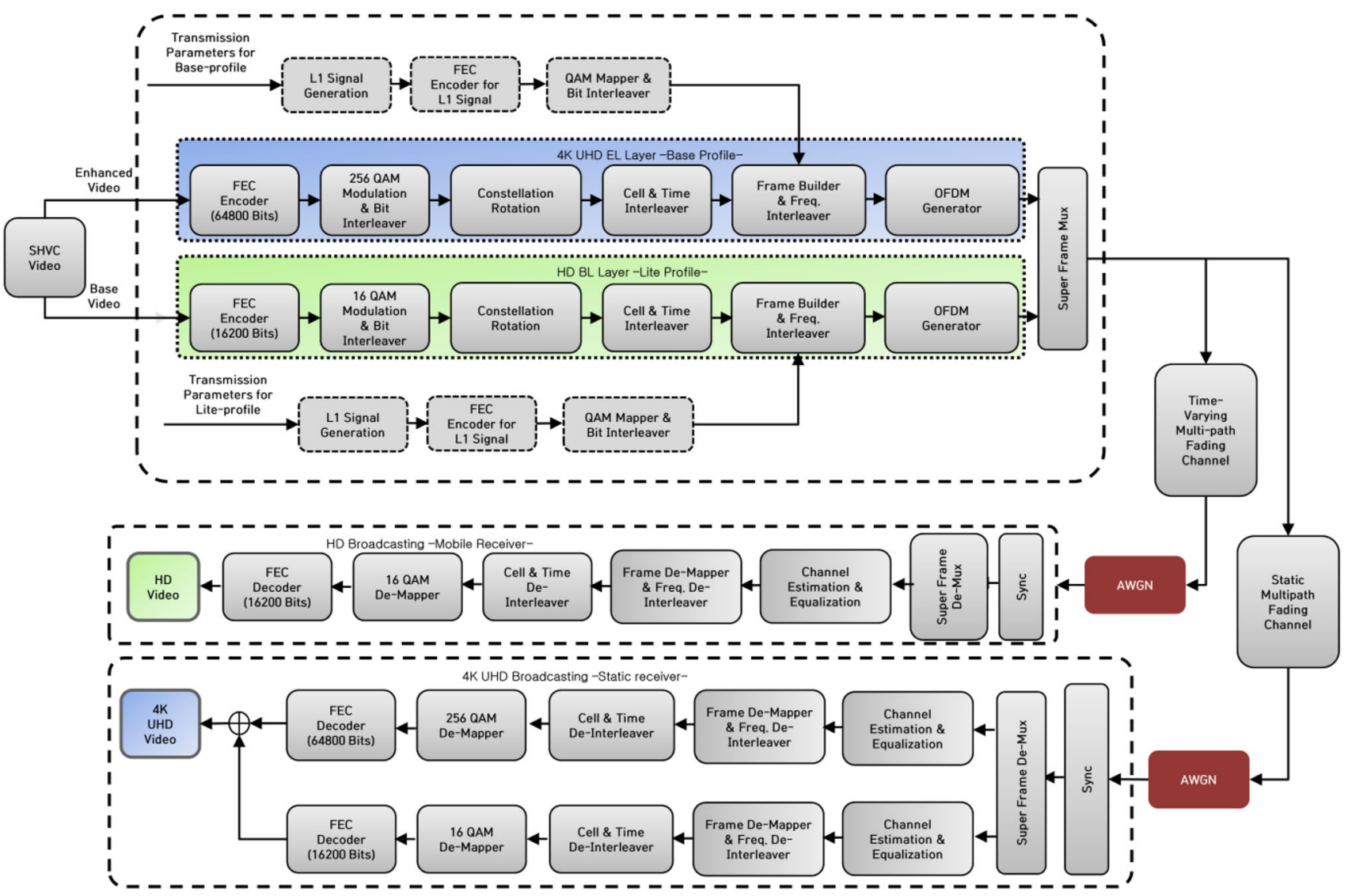

Fig. 12 Structure of terrestrial 4K UHD and HD convergence broadcasting transmission systems through a single channel by employing FEF multiplexing technique

Table 10 Requirements for designing the available transmission parameters for $6 \mathrm{MHz}$ bandwidth

\begin{tabular}{ll}
\hline 4K UHD EL data portion in a frame & $60 \%$ \\
HD BL data portion in a frame & $40 \%$ \\
Maximum length of a frame & $250 \mathrm{~ms} \mathrm{[8]}$ \\
Elementary period $T$ for $6 \mathrm{MHz}$ bandwidth & $7 / 48 \mu \mathrm{s} \mathrm{[8]}$
\end{tabular}

Note: If extended transmission mode is available by FFT size, extended transmission mode is applied

UHD EL data and 16 QAM for HD BL data with detailed parameters in Table 11. A channel environment for 4K UHD EL is a static multipath channel with a long delay path. Thus, for 4K UHD ELPs (enhanced layer profiles) of all transmission parameters, the FFT size is 32,768 , which is the largest; the CP size is $1 / 128$, which is the smallest; the code rate is $5 / 6$, which is the highest; the pilot pattern is PP7 to maximize the data transmission rate. Although the $\mathrm{CP}$ size is $1 / 128$, the FFT size is 32,768 , and the guard interval is $32,768 / 128 \times T=32.33 \mu \mathrm{s}$, which is sufficiently long. On the contrary, in HD base layer profiles (BLPs), the FFT size is smaller and the $\mathrm{CP}$ size is longer than in 4K UHD EL profiles to cope with the fast-fading multipath channel, and code rates are also lower than in 4K UHD EL. In addition, PP 2, 4, and 5, which transmit more pilots than PP 6, 7 , and 8 , are selected. The FEF interval for 4K UHD ELP, FEF_INTERVAL $_{4 K}$, is set to 3 , and the FEF interval for HD ELP, FEF INTERVAL ${ }_{H D}$, is set to 1 . Note that each Base and Lite superframe must be composed of more than two Base or Lite transmission frames [8]. Thus, a 4K UHD layer superframe consists of three successive 4K UHD transmission frames and a consecutive HD transmission frame, and an HD layer superframe consists of two consecutive portions of one HD transmission frame and three successive 4K UHD transmission frames.

To calculate the transmission data rate from the transmission parameters, the FFT and CP size, the number of data OFDM symbols, the number of total carriers and active carriers depending on the PP, and the FEF interval for each layer profile should be considered. Figures 13 and 14 show the structure of a superframe and the transmission frames for each layer profile on a No. 2 transmission parameter in Table 11. The number of subcarriers of the P1 symbol, $K_{p 1}$, for each layer transmission frame is fixed as 2048 regardless of the FFT size. The FFT size for $4 \mathrm{~K}$ UHD ELP, $K_{\mathrm{FFT}-4 \mathrm{~K}}$, is 32,768, and the number of P2 symbols for 4K UHD ELP, 
Table 11 Available transmission parameters for $6 \mathrm{MHz}$ bandwidth by employing FEF multiplexing technique (4K UHD layer: 256 QAM, HD layer: 16 QAM)

\begin{tabular}{|c|c|c|c|c|c|c|c|c|}
\hline \multirow[t]{2}{*}{ Parameter } & \multicolumn{2}{|l|}{1} & \multicolumn{2}{|l|}{$\underline{2}$} & \multicolumn{2}{|l|}{3} & \multicolumn{2}{|l|}{4} \\
\hline & Base $^{\mathrm{a}}$ & Lite $^{\mathrm{b}}$ & Base $^{\mathrm{a}}$ & Lite $^{\mathrm{b}}$ & Base $^{\mathrm{a}}$ & Lite $^{b}$ & Base $^{\mathrm{a}}$ & Lite $^{\mathrm{b}}$ \\
\hline Total bit rate (bps) & $25,093,671$ & & $24,996,827$ & & $24,295,492$ & & $24,138,097$ & \\
\hline Bit rate (bps) & $19,065,495$ & $6,028,176$ & $18,912,877$ & $6,083,948$ & $18,325,594$ & $5,969,898$ & $18,161,477$ & $5,976,619$ \\
\hline Code rate & $5 / 6$ & $3 / 4$ & $5 / 6$ & $2 / 3$ & $5 / 6$ & $3 / 5$ & $5 / 6$ & $3 / 5$ \\
\hline FFT size & 32,768 & 4096 & 32,768 & 4096 & 32,768 & 4096 & 32,768 & 8192 \\
\hline $\mathrm{CP}$ size & $1 / 128$ & $1 / 8$ & $1 / 128$ & $1 / 16$ & $1 / 128$ & $1 / 32$ & $1 / 128$ & $19 / 256$ \\
\hline Pilot pattern & PP7 & PP2 & PP7 & PP4 & PP7 & PP7 & PP7 & PP5 \\
\hline Num. of syms ${ }^{c}$ & 15 & 290 & 14 & 290 & 13 & 295 & 17 & 290 \\
\hline Frame length (ms) & 77.3 & 197.8 & 72.5 & 186.8 & 67.6 & 184.4 & 86.9 & 164.8 \\
\hline FEF interval & 3 & 1 & 3 & 1 & 3 & 1 & 3 & 1 \\
\hline
\end{tabular}

a 4K UHD EL (256 QAM)—Base profile, extended mode

${ }^{\mathrm{b}} \mathrm{HD}$ BL (16 QAM)-Lite profile

${ }^{\mathrm{c}}$ Number of data OFDM symbols in a frame

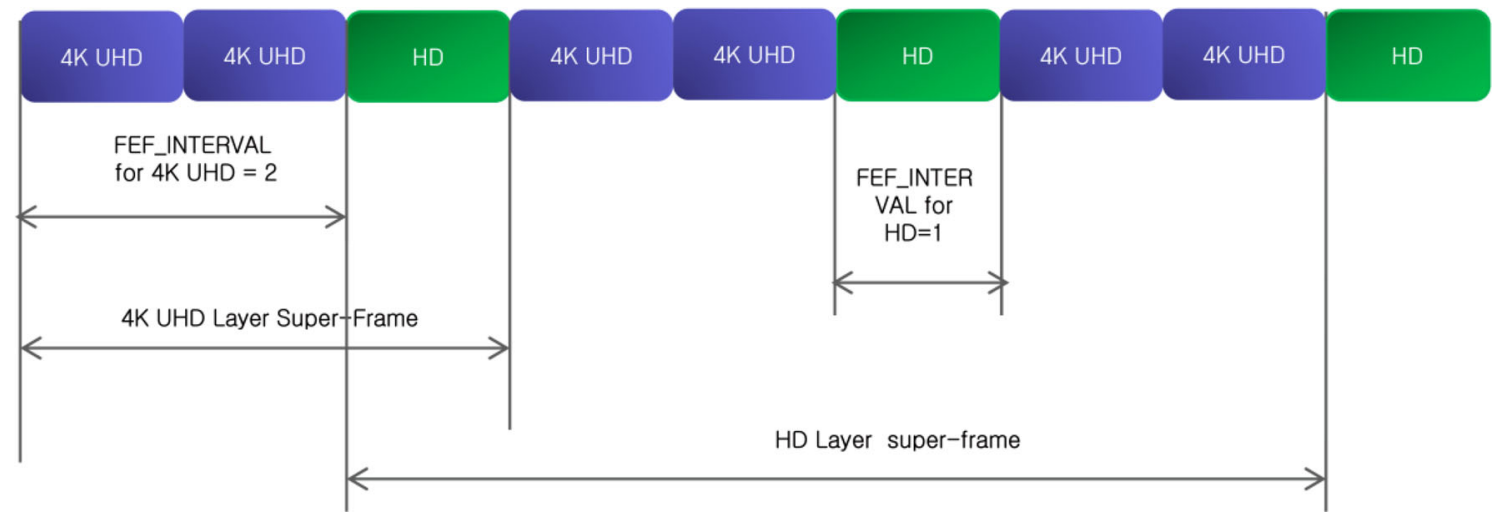

Fig. 13 A superframe structure depending on No. 2 transmission parameter in Table 11

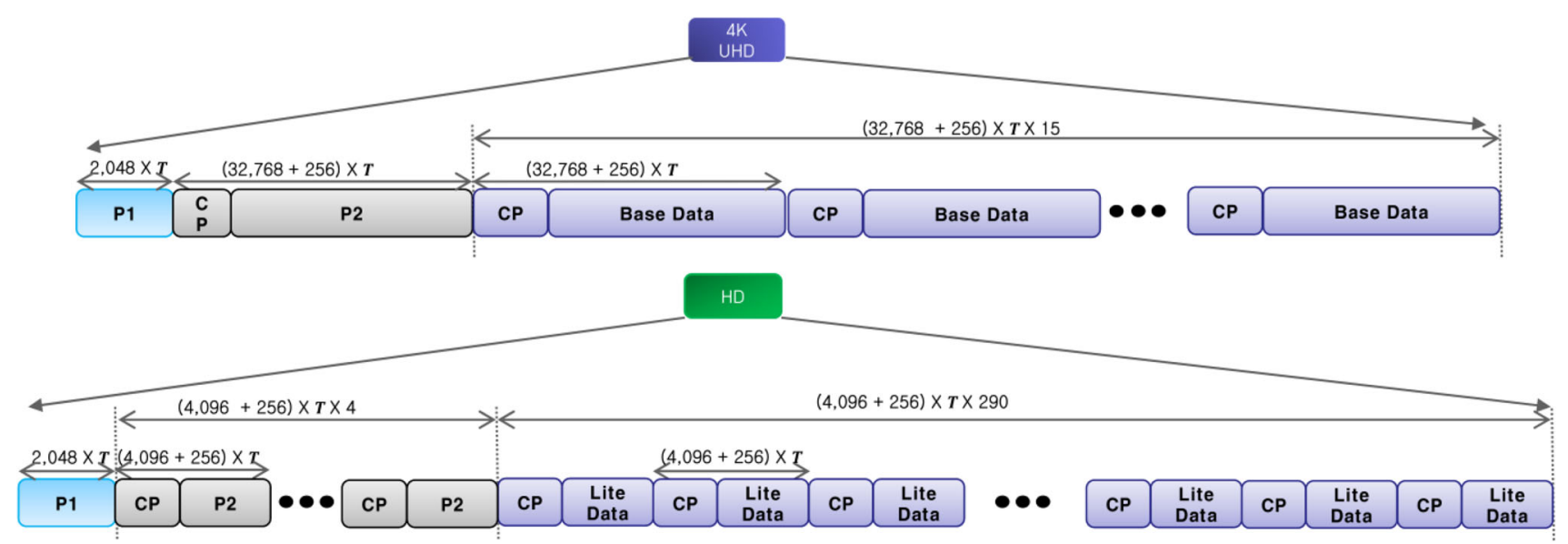

Fig. 14 A transmission frame structure depending on No. 2 transmission parameter in Table 11 
$N_{\mathrm{p} 2-4 \mathrm{~K}}$, is 1 according to Table 2 . The CP size is $1 / 128$, and the number of data OFDM symbols in a 4K UHD transmission frame, $N_{\text {data- } 4 \mathrm{~K}}$, is 14 . Thus, the number of total subcarriers in a $4 \mathrm{~K}$ UHD transmission frame, $K_{\text {frame- } 4 \mathrm{~K}}$, is calculated as in (1).

$$
\begin{aligned}
K_{\text {frame- } 4 \mathrm{~K}}= & K_{P 1}+\left(N_{P 2-4 \mathrm{~K}}\right. \\
& \left.+N_{\text {data- } 4 \mathrm{~K}}\right)\left(K_{\mathrm{FFT}-4 \mathrm{~K}}+\frac{K_{\mathrm{FFT}-4 \mathrm{~K}}}{128}\right) \\
= & 2048 \\
& +(1+14) \times\left(32,768+\frac{32,768}{128}\right) \\
= & 497,408 .
\end{aligned}
$$

For HD BLP, the FFT size, $K_{\mathrm{FFT}-\mathrm{HD}}$, is 4096 ; the number of P2 symbols, $N_{p 2-\mathrm{HD}}$, is 4 ; the CP size is $1 / 16$; the number of data OFDM symbols in an HD transmission frame, $N_{\text {data-HD, }}$ is 290. Therefore, the number of total subcarriers in an HD transmission frame, $K_{\text {frame-HD, }}$ can be written as in (2).

$$
\begin{aligned}
K_{\text {frame-HD }}= & K_{P 1}+\left(N_{P 2-\mathrm{HD}}\right. \\
& \left.+N_{\text {data-HD }}\right)\left(K_{\mathrm{FFT}-\mathrm{HD}}+\frac{K_{\mathrm{FFT}-\mathrm{HD}}}{16}\right) \\
= & 2048 \\
& +(4+290) \times\left(4096+\frac{4096}{16}\right) \\
= & 1,21,536 .
\end{aligned}
$$

A 4K UHD layer superframe consists of three successive 4K UHD transmission frames and a consecutive HD transmission frame. Thus, the number of total subcarriers in a $4 \mathrm{~K}$ UHD layer superframe, $K_{\mathrm{SF}}$, is written as in (3) by applying an elementary period $(T)$, and the length of a $4 \mathrm{~K}$ UHD layer superframe, $T_{\mathrm{SF}}$, is written as in (4).

$$
\begin{aligned}
K_{\mathrm{SF}} & =K_{\text {frame- } 4 \mathrm{~K}} \times 3+K_{\text {frame-HD }} \times 1 \\
& =497,408 \times 3+1,281,536 \times 1=2,773,760 . \\
T_{\mathrm{SF}} & =K_{\mathrm{SF}} \times T=2,773,760 \times\left(7 / 48 \times 10^{-6} \mathrm{~s}\right) \\
& \cong 404.506667 \mathrm{~ms} .
\end{aligned}
$$

For 4K UHD ELP, a frame closing symbol, which transmits more pilots than a normal data OFDM symbol and is the last data OFDM symbol in a transmission frame, is not used by a combination of the FFT size and a PP as in Table 64 of [8]. The number of active subcarriers in a data OFDM symbol for $4 \mathrm{~K}$ UHD ELP, $C_{\text {data-4K }}$, is 27,404 owing to usage of PP7 as in Table 48 of [8]. 3. For HD BLP, a frame closing symbol

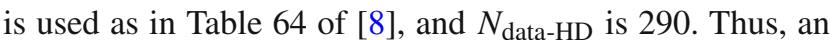
HD layer transmission frame consists of 289 normal data OFDM symbols and 1 frame closing symbol. The number of active subcarriers in a data OFDM symbol for HD BLP, $C_{\text {data-HD, }}$, is 3234 owing to usage of PP4 as in Table 48 of
[8], and the number of active subcarriers in a frame closing symbol, $C_{\mathrm{fc}-\mathrm{HD}}$, is 2831 as in Table 50 of [8]. Therefore, in a $4 \mathrm{~K}$ UHD layer superframe, the number of total active subcarriers for $4 \mathrm{~K}$ UHD ELP, $C_{\text {frame- } 4 \mathrm{~K}}$, is calculated as in (5) and the number of total active subcarriers for HD ELP,

\begin{tabular}{|c|c|}
\hline Channel environment & $\begin{array}{l}\text { AWGN, Brazil-D, and TU-6 } \\
\text { channel }\end{array}$ \\
\hline $\begin{array}{l}\text { Channel estimation method using } \\
\text { pilot symbol }\end{array}$ & Least-square method [16] \\
\hline $\begin{array}{l}\text { Channel interpolation method in } \\
\text { frequency domain }\end{array}$ & $\begin{array}{l}\text { Cubic-spline interpolation method } \\
\text { [17] (the number of pilot symbols } \\
\text { for interpolation: } 12 \text { symbols) }\end{array}$ \\
\hline $\begin{array}{l}\text { Channel interpolation method in } \\
\text { time domain }\end{array}$ & Linear interpolation [18] \\
\hline Center frequency & $\begin{array}{l}476 \mathrm{MHz} \text { (digital TV channel No. } \\
14 \text { in Korea) }\end{array}$ \\
\hline
\end{tabular}
$C_{\text {frame-HD, }}$ is calculated as in (6).

$$
\begin{aligned}
C_{\text {frame- } 4 \mathrm{~K}}= & C_{\text {data-4K }} \times N_{\text {data-4K }} \\
& \times \text { FEF_INTERVAL } 4 K_{-} \\
= & 27,404 \times 14 \times 3=1,150,968 . \\
C_{\text {frame-HD }}= & \left\{\left(C_{\text {data-HD }} \times\left(N_{\text {data-HD }}-1\right)\right)\right. \\
& \left.\left.+\left(C_{\text {fc-HD }} \times 1\right)\right)\right\} \times \text { FEF_INTERVAL } H D \\
= & \{(3234 \times 289)+(2831 \times 1)\} \times 1 \\
= & 937,457 .
\end{aligned}
$$

From (3) to (6), for a duration of approximately $404 \mathrm{ms,}$ we know that 4K UHD EL data are transmitted by $1,150,968$ subcarriers, and HD BL data are transmitted by 937,457 subcarriers. Thus, the number of transmitted active subcarriers for $4 \mathrm{~K}$ UHD ELP per second, $C_{\text {frame- } 4 \mathrm{~K} / \mathrm{s}}$, is calculated as in (7), and the number of transmitted active subcarriers for HD BLP per second, $C_{\text {frame-HD/s }}$, is calculated as in (8), respectively.

Table 12 Computer simulation environmental conditions

Table 13 TU-6 channel profile

\begin{tabular}{llcl}
\hline Tap number & Delay $(\mu \mathrm{s})$ & Power $(\mathrm{dB})$ & Fading model \\
\hline 1 & 0.0 & -3.0 & Rayleigh \\
2 & 0.2 & 0.0 & Rayleigh \\
3 & 0.5 & -2.0 & Rayleigh \\
4 & 1.6 & -6.0 & Rayleigh \\
5 & 2.3 & -8.0 & Rayleigh \\
6 & 5.0 & -10.0 & Rayleigh \\
\hline
\end{tabular}


Table 14 Brazil-D channel profile

\begin{tabular}{llll}
\hline Tap number & Delay $(\mu \mathrm{s})$ & Power $(\mathrm{dB})$ & Fading model \\
\hline 1 & 0.15 & -0.1 & Static \\
2 & 0.63 & -3.8 & Static \\
3 & 2.22 & -2.6 & Static \\
4 & 3.05 & -1.3 & Static \\
5 & 5.86 & 0 & Static \\
6 & 5.93 & -2.8 & Static \\
\hline
\end{tabular}

$$
\begin{aligned}
C_{\text {frame- } 4 \mathrm{~K} / \mathrm{s}} & =C_{\text {frame- } 4 \mathrm{~K}} / T_{\mathrm{SF}} \\
& =1,150,968 / 0.404506667 \\
& \cong 2,845,362 \text { subcarriers } / \mathrm{s} . \\
C_{\text {frame-HD } / \mathrm{s}} & =C_{\text {frame- } \mathrm{HD} /} / T_{\mathrm{SF}} \\
& =937,457 / 0.404506667 \\
& \cong 2,317,532 \text { subcarriers } / \mathrm{s} .
\end{aligned}
$$

Using (7), the data rate of $4 \mathrm{~K}$ UHD ELP, $R_{4 \mathrm{KUHD}}$, can be calculated by employing 256 QAM constellation and 5/6 code rate as,

$$
\begin{aligned}
R_{4 \mathrm{KUHD}} & =C_{\text {frame- } 4 \mathrm{~K} / \mathrm{s}} \times 8 \times \frac{53,840}{64,800} \\
& \cong 18,912,877 \mathrm{bps} .
\end{aligned}
$$

Similarly, using (8), the data rate of HD BLP, $R_{\mathrm{HD}}$, can be calculated by employing 16 QAM constellation and $2 / 3$ code rate as,

$$
\begin{aligned}
R_{\mathrm{HD}} & =C_{\text {frame- } \mathrm{HD} / \mathrm{s}} \times 4 \times \frac{10,632}{16,200} \\
& \cong 6,083,948 \mathrm{bps} .
\end{aligned}
$$

Using the available transmission parameters, the reception performance was analyzed using computer simulations under the environmental conditions shown in Table 12. The TU-6 channel profile listed in Table 13 is employed for computer simulations, and the Rayleigh fading channel model is implemented by the method described in [19]. In Table 14, the Brazil-D channel profile employed for the computer simulations is listed, and a static fading channel is assumed. Note that the decoding of the received system information and receiver synchronization was assumed to be ideal.

Figures 15, 16, 17 and 18 show the bit error rate (BER) performance versus the carrier-to-noise ratio (CNR) of transmission parameter Nos. 1-4 in Table 11 for $6 \mathrm{MHz}$ bandwidth under AWGN, Brazil-D channel for 4K UHD ELP, and TU-6 channel for HD BLP. The TOV performance of the available transmission parameters is summarized in Table 15. The TOV data indicate both a reception performance of less than $3 \times 10^{-6}$ BER [20] and the limit of reliable broadcasting performance at the transmission system level. In general, a
Fig. 15 BER performances of 4K UHD and HD layer transmission (No. 1 transmission parameter, $6 \mathrm{MHz}$ bandwidth)

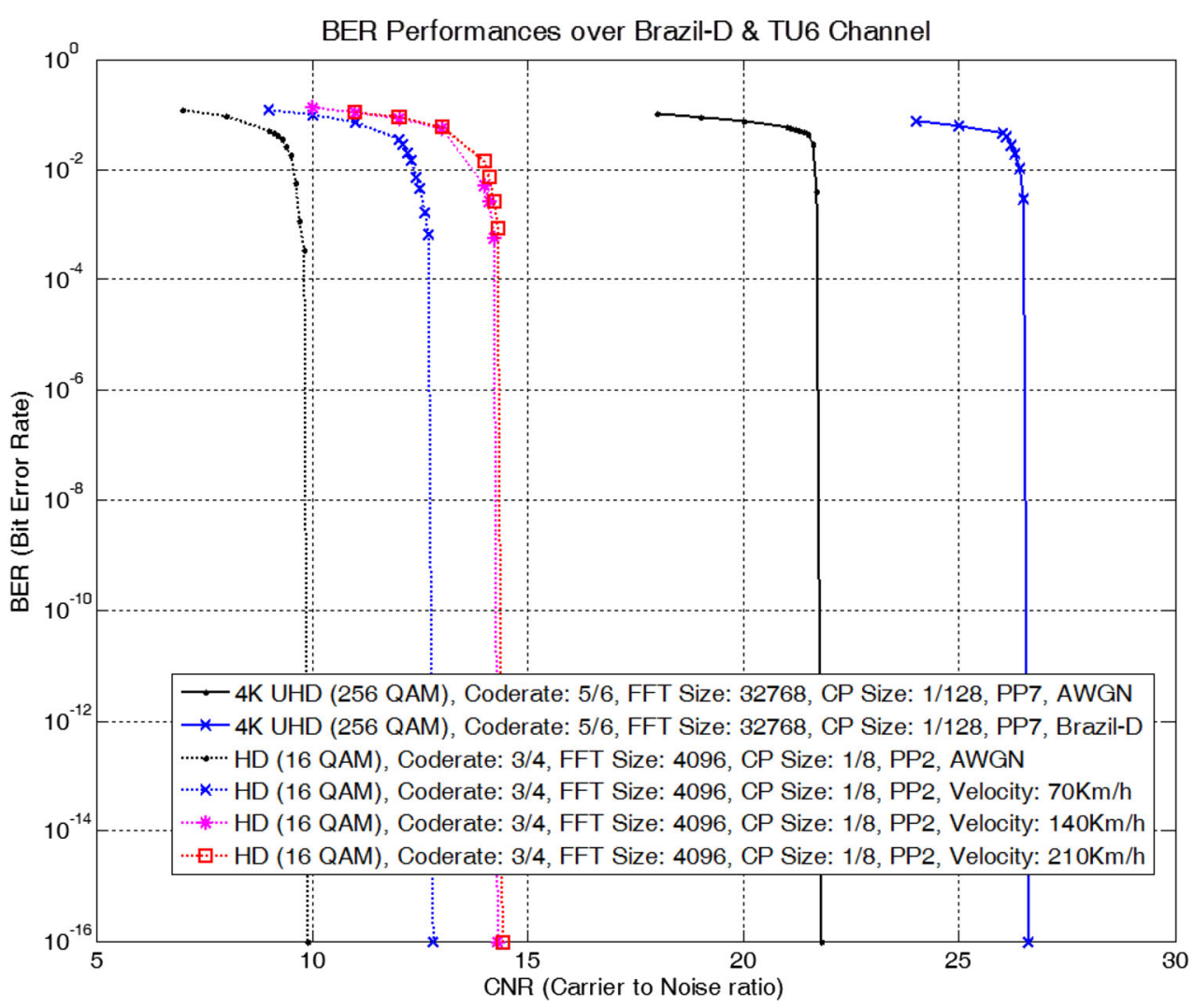


Fig. 16 BER performances of 4K UHD and HD layer transmission (No. 2 transmission parameter, $6 \mathrm{MHz}$ bandwidth)
Fig. 17 BER performances of 4K UHD and HD layer transmission (No. 3 transmission parameter, $6 \mathrm{MHz}$ bandwidth)
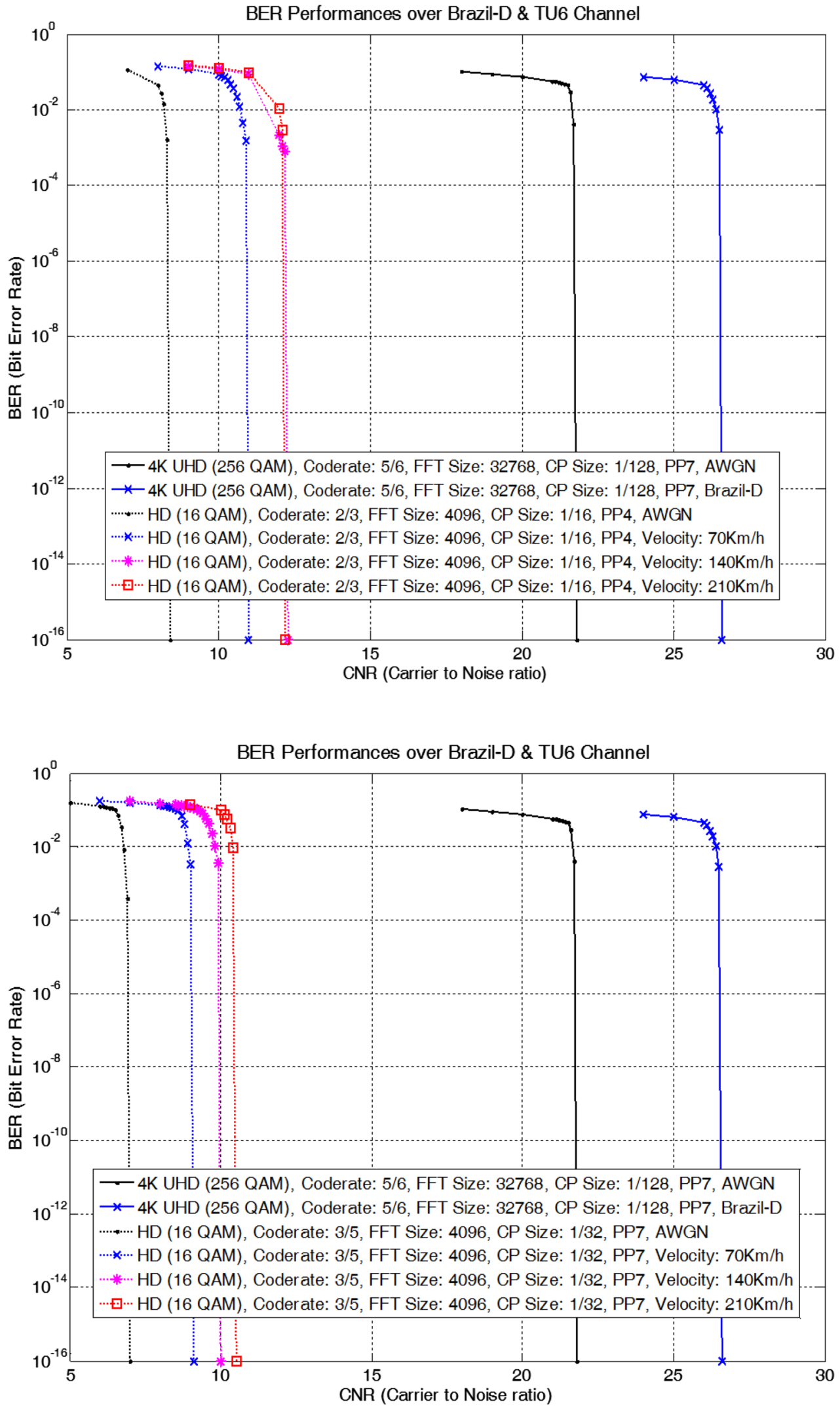
Fig. 18 BER performances of 4K UHD and HD layer transmission (No. 4 transmission parameter, $6 \mathrm{MHz}$ bandwidth)

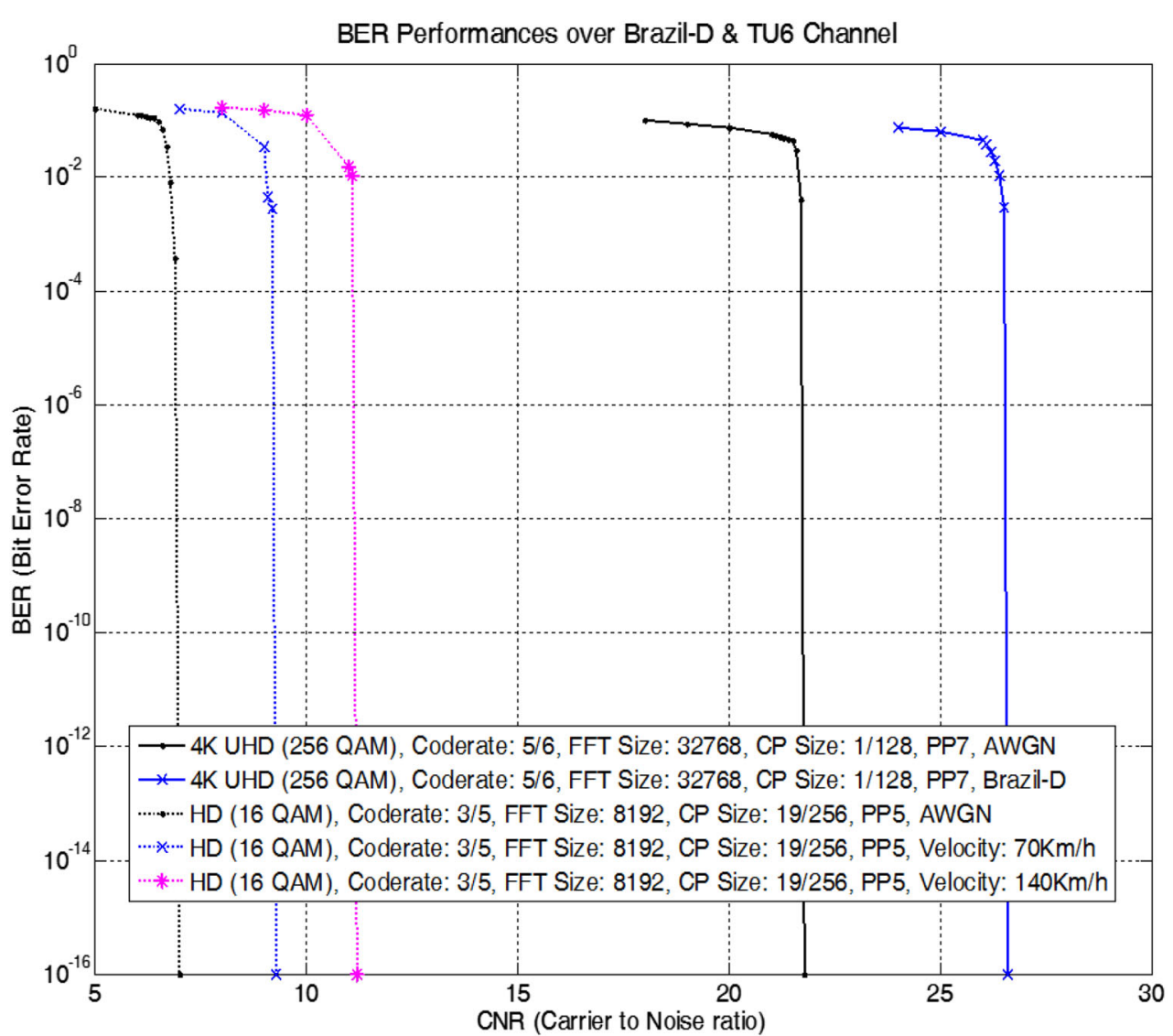

Table 15 TOV performances of available transmission parameters for $6 \mathrm{MHz}$ bandwidth

\begin{tabular}{|c|c|c|c|c|}
\hline \multirow[t]{2}{*}{ Parameter no } & \multicolumn{2}{|l|}{ 4K UHD ELP } & \multirow[b]{2}{*}{ Static Brazil-D channel } & \multirow[b]{2}{*}{$(\mathrm{dB})$} \\
\hline & AWGN & $(\mathrm{dB})$ & & \\
\hline $1-4$ & 21.8 & & 26.6 & \\
\hline \multirow[t]{2}{*}{ Parameter no } & \multicolumn{4}{|l|}{ HD BLP } \\
\hline & AWGN (dB) & TU-6 $(70 \mathrm{~km} / \mathrm{h})(\mathrm{dB})$ & TU-6 (140 km/h) (dB) & TU-6 $(210 \mathrm{~km} / \mathrm{h})(\mathrm{dB})$ \\
\hline 1 & 9.9 & 12.8 & 14.3 & 14.4 \\
\hline 2 & 8.4 & 11.0 & 12.3 & 12.2 \\
\hline 3 & 7.0 & 9.1 & 10.0 & 10.5 \\
\hline 4 & 7.0 & 9.3 & 11.2 & $X$ \\
\hline
\end{tabular}

return path does not exist in the broadcasting environment. Thus, only when the CNR is greater than the TOV can seamless broadcasting services be provided to viewers. For the TOV of available transmission parameter No. 2 in Table 11, reliable HD broadcasting can be achieved when the velocity of the receiver is $210 \mathrm{~km} / \mathrm{h}$ and the CNR is greater than 12.2 $\mathrm{dB}$.

Under a static Brazil-D channel, the TOV of 4K UHD ELP is $26.6 \mathrm{~dB}$, and a $4.8 \mathrm{~dB}$ performance degradation occurred
Table 16 Requirements for designing the available transmission parameters for $8 \mathrm{MHz}$ bandwidth

\begin{tabular}{ll}
\hline 4K UHD EL data portion in a frame & $60 \%$ \\
HD BL data portion in a frame & $40 \%$ \\
Maximum length of a frame & $250 \mathrm{~ms}[8]$ \\
Elementary period $T$ for $6 \mathrm{MHz}$ bandwidth & $7 / 64 \mu \mathrm{s} \mathrm{[8]}$
\end{tabular}

Note: If extended transmission mode is available by FFT size, extended transmission mode is applied 
Table 17 Available transmission parameters for $8 \mathrm{MHz}$ bandwidth

\begin{tabular}{|c|c|c|c|c|c|c|c|c|}
\hline \multirow[t]{2}{*}{ Parameter } & \multicolumn{2}{|l|}{1} & \multicolumn{2}{|l|}{2} & \multicolumn{2}{|l|}{3} & \multicolumn{2}{|l|}{4} \\
\hline & Base $^{\mathrm{a}}$ & Lite $^{b}$ & Base $^{a}$ & Lite $^{b}$ & Base $^{\mathrm{a}}$ & Lite $^{b}$ & Base $^{a}$ & Lite $^{b}$ \\
\hline Total bit rate (bps) & $29,012,926$ & & $29,523,782$ & & $29,839,890$ & & $29,870,920$ & \\
\hline Bit rate (bps) & $21,325,164$ & 7,347,699 & $21,994,129$ & $7,178,923$ & $22,357,368$ & $7,125,997$ & $22,369,552$ & $7,144,651$ \\
\hline Code rate & $4 / 5$ & $3 / 5$ & $4 / 5$ & $2 / 3$ & $4 / 5$ & $3 / 5$ & $4 / 5$ & $2 / 3$ \\
\hline FFT size & 32,768 & 2048 & 32,768 & 2048 & 32,768 & 4096 & 32,768 & 4096 \\
\hline CP size & $1 / 128$ & $1 / 8$ & $1 / 128$ & $1 / 4$ & $1 / 128$ & $1 / 8$ & $1 / 128$ & $1 / 4$ \\
\hline Pilot pattern & PP7 & PP2 & PP7 & PP1 & PP7 & PP2 & PP7 & PP1 \\
\hline Num. of syms ${ }^{c}$ & 12 & 600 & 14 & 600 & 13 & 298 & 14 & 290 \\
\hline Frame length (ms) & 47.18 & 153.4 & 54.40 & 170.4 & 50.79 & 152.4 & 54.4 & 164.8 \\
\hline FEF interval & 3 & 1 & 3 & 1 & 3 & 1 & 3 & 1 \\
\hline
\end{tabular}

a 4K UHD enhanced layer (256 QAM) - Base profile, extended mode

${ }^{\mathrm{b}} \mathrm{HD}$ base layer (16 QAM)-Lite profile

${ }^{c}$ Number of data OFDM symbols in a frame

with the multipath channel compared to the performance under AWGN. However, reliable reception of 4K UHD EL data can be achieved under indoor environments, which is assumed in the static Brazil-D channel. For HD BLP, the TOVs are in the range of 9.1-14.4 $\mathrm{dB}$ when the velocity of the receiver is 70,140 , and $210 \mathrm{~km} / \mathrm{h}$, and reliable reception of HD BL data can be achieved under the fast-fading mobile channel. However, for No. 4 transmission parameter, proper reception of HD BL data when the velocity of the receiver is $210 \mathrm{~km} / \mathrm{h}$ is not possible owing to the limitation of the large FFT size $(8,192)$. It is noteworthy that the TOV of No. 3 transmission parameter is lower than that of No. 1 and No. 2 transmission parameters. When the FFT sizes of No. 1-3 transmission parameters are the same, the TOV performance of No. 3 transmission parameter can be improved by employing PP7, which transmits smaller pilots than PP2 or 4, and by lowering the code rate to $3 / 5$.

In Europe, $8 \mathrm{MHz}$ bandwidth is allocated for terrestrial channels, and the extension of this bandwidth is currently being discussed among governments, broadcasters, and mobile service providers globally. In addition, previously in Sect. 4.2, we assumed the data rate for a $6 \mathrm{MHz}$ bandwidth channel is decreased by $15 \%$ compared to the estimated data rate in Sect. 2.2 to overcome the limit of $6 \mathrm{MHz}$ bandwidth. However, the estimated data rate in Sect. 2.2 can be applied to the $8 \mathrm{MHz}$ bandwidth channel, and high-quality $4 \mathrm{~K}$ UHD and HD broadcasting services can be provided.

Thus, the available transmission parameters were redesigned for the $8 \mathrm{MHz}$ bandwidth channel. The available parameters for $8 \mathrm{MHz}$ bandwidth that meet the requirements in Table 16 are listed in Table 17.

Every supported constellation point in the DVB-T2 systems was considered for 4K UHD ELP and HD BLP. However, similar to the case of $6 \mathrm{MHz}$ bandwidth, the required rate could be met when 256 QAM was used for
4K UHD ELP and 16 QAM for the HD ELP. For 4K UHD ELP of all transmission parameters, the FFT size is set to 32,768 which is the largest; the CP size is set to $1 / 128$, which is the smallest; the pilot pattern is set to PP7, the same as for $6 \mathrm{MHz}$ bandwidth, but the code rate can be lowered from $5 / 6$ to $4 / 5$ by extending the bandwidth to $8 \mathrm{MHz}$. In addition, the maximum code rate was restricted to $2 / 3$, and the pilot modes $3,4,5,6$, and 7 were excluded to take into consideration HD ELP under the mobile channel environment by extending the bandwidth to $8 \mathrm{MHz}$. For No. 1 and 2 transmission parameters, by extending the bandwidth to $8 \mathrm{MHz}$, the FFT size can be set to 2048 and the CP size to $1 / 8$ and $1 / 4$, which is the long guard interval. Similarly, for No. 3 and 4 transmission parameters, the FFT size can be set to 4,096 and the CP size to $1 / 8$ and $1 / 4$, which is the long guard interval. In No. 2 and 4 transmission parameters, each $\mathrm{CP}$ size is set to be longer than each CP size of No. 1 and 3 transmission parameters by increasing each code rate.

Using the available transmission parameters, the reception performance was analyzed using computer simulations under the environmental conditions given in Table 12. Note that the decoding of the received system information and receiver synchronization were assumed to be ideal.

Figures 19, 20, 21 and 22 show the BER performance versus CNR of transmission parameters No. 1-4 in Table 17 for $8 \mathrm{MHz}$ bandwidth under AWGN, Brazil-D channel for $4 \mathrm{~K}$ UHD ELP, and TU-6 channel for HD BLP. The TOV performance of the available transmission parameters is summarized in Table 18.

Under a static Brazil-D channel, the TOV of 4K UHD ELP is $20.9 \mathrm{~dB}$, and a performance degradation of $4.3 \mathrm{~dB}$ occurred with the multipath channel compared to the performance under AWGN. However, reliable reception of $4 \mathrm{~K}$ UHD EL data can be achieved under indoor environments for $8 \mathrm{MHz}$ bandwidth as well as for $6 \mathrm{MHz}$ bandwidth. For 
Fig. 19 BER performances of 4K UHD and HD layer transmission (No. 1 transmission parameter, $8 \mathrm{MHz}$ bandwidth)

Fig. 20 BER performances of 4K UHD and HD layer transmission (No. 2 transmission parameter, $8 \mathrm{MHz}$ bandwidth)
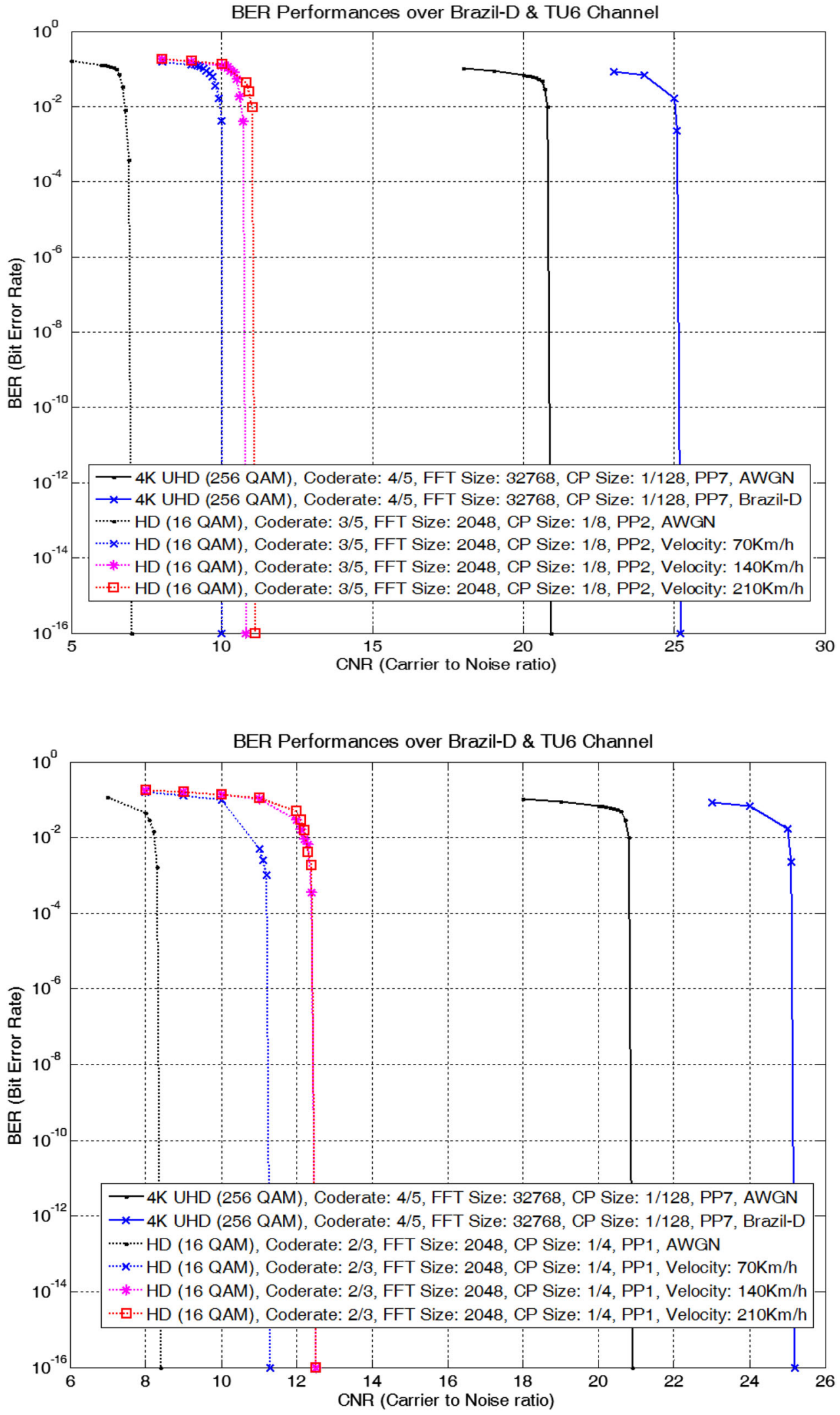
Fig. 21 BER performances of 4K UHD and HD layer transmission (No. 3 transmission parameter, $8 \mathrm{MHz}$ bandwidth)
Fig. 22 BER performances of 4K UHD and HD layer transmission (No. 4 transmission parameter, $8 \mathrm{MHz}$ bandwidth)
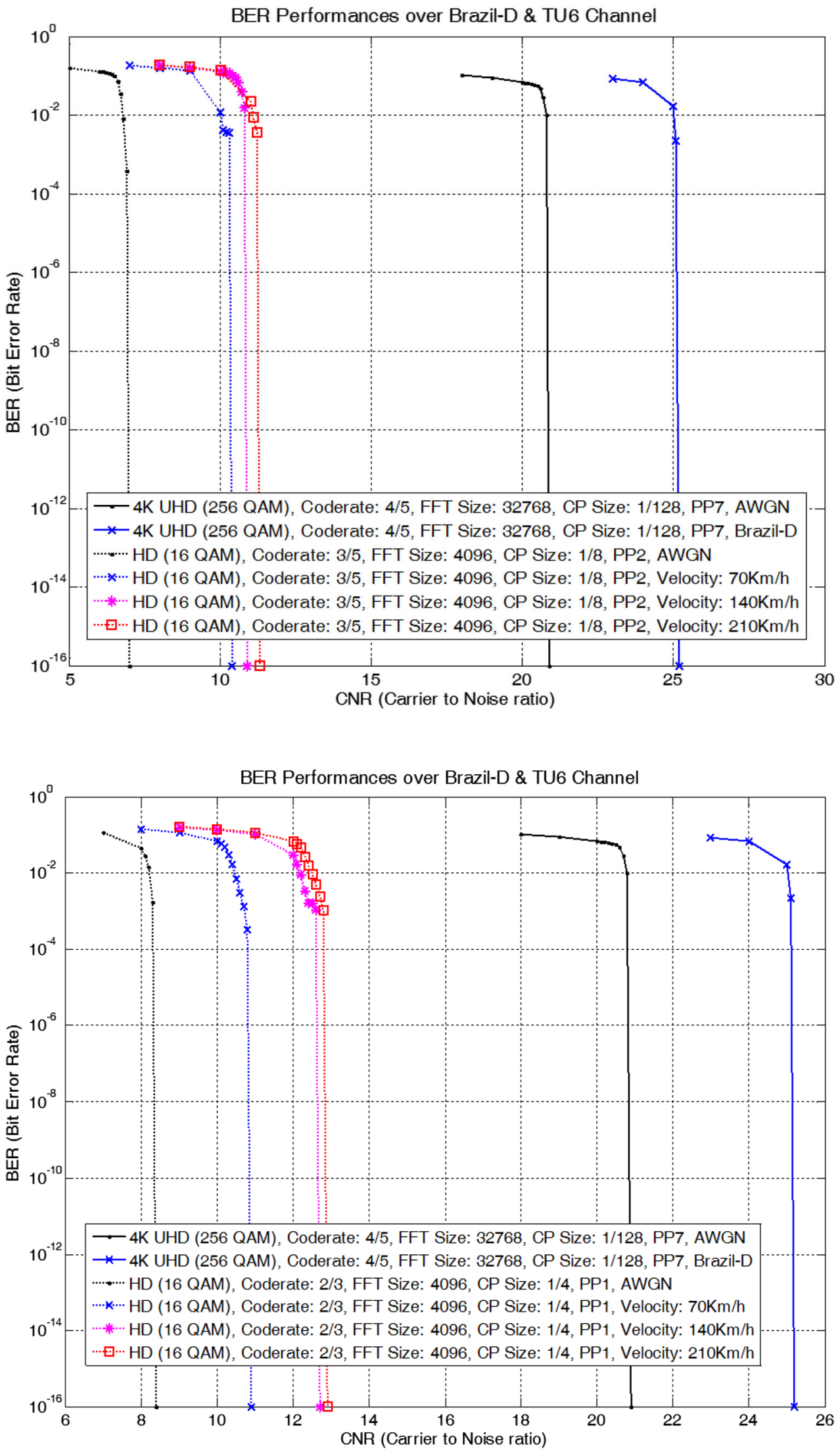
Table 18 TOV performances of available transmission parameters for $8 \mathrm{MHz}$ bandwidth

\begin{tabular}{|c|c|c|c|c|}
\hline \multirow[t]{2}{*}{ Parameter no. } & \multicolumn{4}{|l|}{$\underline{4 K}$ UHD ELP } \\
\hline & AWGN & $(\mathrm{dB})$ & Static Brazil-D channel & $(\mathrm{dB})$ \\
\hline $1 \sim 4$ & $20.9 \mathrm{~dB}$ & & $25.2 \mathrm{~dB}$ & \\
\hline \multirow[t]{2}{*}{ Parameter No. } & \multicolumn{2}{|l|}{ HD BLP } & & \\
\hline & AWGN (dB) & TU-6 $(70 \mathrm{~km} / \mathrm{h})(\mathrm{dB})$ & TU-6 (140 km/h) (dB) & TU-6 $(210 \mathrm{~km} / \mathrm{h})(\mathrm{dB})$ \\
\hline 1 & 7.0 & 10.1 & 10.8 & 11.1 \\
\hline 2 & 8.4 & 11.3 & 12.5 & 12.5 \\
\hline 3 & 7.0 & 10.4 & 10.9 & 11.3 \\
\hline 4 & 8.4 & 10.9 & 12.7 & 12.9 \\
\hline
\end{tabular}

HD BLP, the TOVs are all in the range 10.1-12.9 $\mathrm{dB}$ when the velocity of the receiver is 70,140 , and $210 \mathrm{~km} / \mathrm{h}$, and reliable reception of $\mathrm{HD}$ BL data can be achieved under the fast-fading mobile channel. For No. 2 and 4 transmission parameters, each $\mathrm{CP}$ size is set to be longer than each $\mathrm{CP}$ size of No. 1 and 3 transmission parameters by increasing each code rate, and the TOV performance for No. 2 and 4 transmission parameters is lower than that for No. 1 and 3 transmission parameters, respectively. The guard intervals of HD BLP for all transmission parameters are enough for the longest delay path of the TU-6 channel. However, in the long delay channel compared to TU-6, the TOV performance for No. 2 and 4 can be higher than that for No. 1 and 3 transmission parameters.

\section{Conclusion}

As a part of a project, which aims to develop convergence broadcasting and monitoring systems that provide optimized and high-quality broadcasting services for both fixed and mobile receivers based on broadcasting and communication networks, the possibility and the performance of a terrestrial fixed 4K UHD \& mobile HD convergence broadcasting service through a single channel employing the FEF multiplexing technique in DVB-T2 systems are examined in this paper. By employing an FEF multiplexing technique instead of M-PLP multiplexing technique, convergence broadcasting, which is appropriate to channel situations of both fixed \& mobile receiver and layered transmission, can be achieved. By computer simulations, it is shown that reliable reception of fixed 4K UHD \& mobile HD convergence broadcasting can be achieved through $6 \& 8 \mathrm{MHz}$ terrestrial channel under targeting channel situations. In addition, when the bandwidth is extended to $8 \mathrm{MHz}$, high-quality convergence broadcasting services can be provided.

By achieving this work, it is shown that commercial level convergence broadcasting through terrestrial broadcasting and communication network can be provided to consumers with the development of associate protocol based on MPEG media transport (MMT), network layer systems, and monitoring systems are finished.

Recently, a layer division multiplexing (LDM) technique is focused as one of the key feature that makes the standard unique of ATSC 3.0 systems. LDM splits the total transmission power into two components (layers) that overlap in frequency (upper and lower layers), and it enables the use of a single RF channel for delivering high-capacity services to fixed receivers and low-complexity robust services to mobile receivers [21]. Thus, for future work, by employing LDM techniques in ATSC 3.0 systems, the possibility and the performance of a terrestrial fixed $4 \mathrm{~K}$ UHD \& mobile HD convergence broadcasting service through a single channel should also be examined.

Acknowledgements This work was supported by ICT R\&D program of MSIP/IITP (R0101-15-0189, Development of the next-generation convergence broadcasting and monitoring systems combined with the networks).

Open Access This article is distributed under the terms of the Creative Commons Attribution 4.0 International License (http://creativecomm ons.org/licenses/by/4.0/), which permits unrestricted use, distribution, and reproduction in any medium, provided you give appropriate credit to the original author(s) and the source, provide a link to the Creative Commons license, and indicate if changes were made.

\section{References}

1. ETSI TS 102796 V1.2.1 (2012) Hybrid broadcast broadband TV, ETSI

2. Malhotra R (2013) Hybrid broadcast broadband TV: the way forward for connected TVs. IEEE Consum Electron Mag 2(33):10-16

3. ATSC, ATSC digital television standard, ATSC Doc.A/53, 1995.9.5

4. ETSI TS 102427 V1.1.1 (2005) Digital audio broadcasting (DAB); data broadcasting-MPEG-2 TS streaming, ETSI

5. ETSI TS 102563 V1.1.1 (2007) Digital audio broadcasting (DAB); transport of advanced audio coding audio, ETSI

6. Oh JG, Won WJ, Lee JS, Kim YH, Paik JH, Kim JT (2015) A study of development of transmission systems for next-generation terrestrial $4 \mathrm{~K}$ UHD and HD convergence broadcasting. EURASIP J Wirel Commun Netw 2015:128 
7. Yu KA, Seo MJ, Paik JH (2014) Design of MMT signaling for streaming service based on SVC in mobile environments. In: 6th international conference of internet (ICONI), Taipei, pp 195-196

8. ETSI EN 302755 v1.3.1 (2011) Digital video broadcasting (DVB); frame structure channel coding and modulation systems for a second generation digital terrestrial television broadcasting system, ETSI

9. Sullivan GJ, Ohm JR, Han WJ, Wiegand T (2012) Overview of the high efficiency video coding (HEVC) standard. IEEE Trans Circ Syst Video Technol 22(12):1649-1668

10. Ye Y, Andrivon P (2014) The scalable extensions of HEVC for ultra-high-definition video delivery. IEEE Multimed 21(3):58-64

11. Schwarz H, Marpe D, Wiegand T (2007) Overview of the scalable video coding extension of the H.264/AVC standard. IEEE Trans Circ Syst Video Technol 17(9):103-120

12. Zou WY, Wu Y (1995) COFDM: an overview. IEEE Trans Broadcast 41(1):1-8

13. Gallager RG (1963) Low density parity-check codes. MIT Press, Cambridge

14. Hocquenghem A (1959) Codes correcteurs d'erreurs. Chiffres $2: 147-156$
15. Bose RC (1960) DKR Chaudhuri. On a class of error correcting binary group codes, information and control 3(1):68-79

16. van de Beek JJ, Edfors O, Sandell M, Wilson SK, Börjesson PO (1995) On channel estimation in OFDM systems. In: Vehicular technology conference (VTC), Chicago, pp 815-819

17. McKinley S, Levine M (2007) Cubic spline interpolation. http:// online.redwoods.cc.ca.us/instruct/darnold/laproj/Fall98/SkyMeg/ proj.pdf

18. ETSI EN 102831 v1.2.1 (2012) Digital video broadcasting (DVB); implementation guidelines for a second generation digital terrestrial television broadcasting system, ETSI

19. Fechtel SA (1993) A novel approach to modelling and efficient simulation of frequency-selective fading channels. IEEE Trans Sel Areas Commun 11(3):422-431

20. Lee YT, Park SI, Kim SW, Ahn CT, Seo JS (2004) ATSC terrestrial digital television broadcasting using single frequency networks. ETRI J 26(2):92-100

21. Cristina R, Jon M, Jon M, Manuel V, Pablo A, Yiyan W, Liang Z, Sung-Ik P, Jae-Young L, Heung Mook K (2016) IEEE LDM core services performance in ATSC 3.0. IEEE Trans Broadcast 62(1):244-252 